\title{
MEASUREMENT OF PLANET MASSES WITH TRANSIT TIMING VARIATIONS DUE TO SYNODIC “CHOPPING” EFFECTS
}

\author{
Katherine M. Deck ${ }^{1}$ and ERIC Agol ${ }^{2}$ \\ ${ }^{1}$ Division of Geological and Planetary Sciences, California Institute of Technology, Pasadena, CA 91101, USA; kdeck@caltech.edu \\ ${ }^{2}$ Department of Astronomy, University of Washington, Box 351580, Seattle, WA 98195, USA; agol@astro.washington.edu \\ Received 2014 October 28; accepted 2015 January 21; published 2015 April 1
}

\begin{abstract}
Gravitational interactions between planets in transiting exoplanetary systems lead to variations in the times of transit that are diagnostic of the planetary masses and the dynamical state of the system. Here we show that synodic "chopping" contributions to these transit timing variations (TTVs) can be used to uniquely measure the masses of planets without full dynamical analyses involving direct integration of the equations of motion. We present simple analytic formulae for the chopping signal, which are valid (generally $<10 \%$ error) for modest eccentricities $e \lesssim 0.1$. Importantly, these formulae primarily depend on the mass of the perturbing planet, and therefore the chopping signal can be used to break the mass/free-eccentricity degeneracy, which can appear for systems near first-order mean motion resonances. Using a harmonic analysis, we apply these TTV formulae to a number of Kepler systems, which had been previously modeled with full dynamical analyses. We show that when chopping is measured, the masses of both planets can be determined uniquely, in agreement with previous results, but without the need for numerical orbit integrations. This demonstrates how mass measurements from TTVs may primarily arise from an observable chopping signal. The formula for chopping can also be used to predict the number of transits and timing precision required for future observations, such as those made by TESS or PLATO, in order to infer planetary masses through analysis of TTVs.
\end{abstract}

Key words: planetary systems

\section{INTRODUCTION}

In a multi-planet system, mutual gravitational interactions between planets lead to deviations from Keplerian orbits. In particular, the instantaneous orbital periods are no longer constant, which in turn implies that transiting planets in multiplanet systems will not transit at a fixed, constant rate. The detection of these changes in the transit rate, or "transit timing variations" (TTVs), was initially recognized as a way to infer the presence of non-transiting planets in systems with at least one other transiting planet (Miralda-Escudé 2002; Schneider 2003; Agol et al. 2005; Holman \& Murray 2005). TTVs have since been used to confirm that a transit light curve signal is due to a planetary transit (e.g., Holman et al. 2010), to constrain planetary orbital elements and measure planetary masses using photometry alone (e.g., Carter et al. 2012), and to detect and characterize non-transiting planets (e.g., Ballard et al. 2011; Nesvorný et al. 2012).

TTV data are most commonly analyzed through inversion, a process through which observed transit times are fit using a model of gravitationally interacting planets in order to determine the system parameters, including planetary masses relative to the mass of the star, as well as orbital elements. ${ }^{3}$ Transiting exoplanets generally have well constrained radii, so measurements of their masses yield information regarding densities, bulk compositions, escape velocities, and gravities. This information in turn can be used to identify promising targets for atmospheric characterization and to constrain planetary formation and dynamical evolution (e.g., Hansen \& Murray 2013). Of the orbital elements, constraints on the planetary eccentricities in particular are necessary to

\footnotetext{
3 The relative frequency of TTVs in different types of systems has also been analyzed (Xie et al. 2014).
}

understand the importance of interaction with the protoplanetary disk, interactions with remnant planetesimals, and tidal dissipation (e.g., Lithwick \& Wu 2012; Batygin \& Morbidelli 2013; Hansen \& Murray 2013, 2014; Mahajan \& Wu 2014).

However, the TTV inversion problem is often complicated by strong nonlinear correlations between parameters in a large dimensional space, and as a result precise planetary mass and orbit measurements can be difficult to make. Many of the Kepler multi-planet systems with partially characterized planetary orbits and masses are those near first-order mean motion resonances, a configuration in which the period ratio of two planets is close to $P_{1} / P_{2} \approx\left(j_{R}-1\right) / j_{R}$, where $j_{R}$ is an integer greater than unity, $P_{1}$ is the period of the inner planet, and $P_{2}$ is the period of the outer planet. This is because, for nearly circular orbits and given planet-to-star mass ratios, TTVs are largest in amplitude near first-order mean motion resonances (e.g., Agol et al. 2005; Holman \& Murray 2005). If the planets are near to, but not in, resonance, then the planets show sinusoidal variations with a period equal to the "superperiod,"

$$
P^{j}=\frac{1}{\left|j_{R} / P_{2}-\left(j_{R}-1\right) / P_{1}\right|}
$$

(Agol et al. 2005; Lithwick et al. 2012). However, the amplitude of this TTV signal depends on both the mass of the perturbing planet and the eccentricity vectors of both planets (Lithwick et al. 2012). This degeneracy can be broken statistically with analyses of a large number of planetary systems (Hadden \& Lithwick 2013) or for systems with very precisely measured transit times; however, in practice it inhibits the measurement of the masses and limits our knowledge of the eccentricities of individual planetary systems. 
In spite of this mass-eccentricity degeneracy (and others ${ }^{4}$ ), it has been possible in some cases to precisely measure the masses of planets using TTVs (e.g., Carter et al. 2012; Nesvorný et al. 2013; Dreizler \& Ofir 2014a; Masuda 2014; Nesvorný et al. 2014). The successful mass measurements in these systems are due to the fact that an additional, independent periodic component of the TTVs, with a timescale other than the super-period, was resolved. Other components of TTVs have amplitudes that depend on the orbital parameters and masses in different ways, and so the measurement of secondary components leads to additional, independent constraints on orbital parameters. In particular, the so-called short-timescale ${ }^{5}$ "chopping" TTV associated with the planetary synodic timescale has been identified as an important feature for unique characterization of systems (Holman et al. 2010; Nesvorný et al. 2013). More recently, Nesvorný \& Vokrouhlický (2014b) studied this chopping TTV to clarify how, despite degeneracies between parameters, the TTV method can be used to measure planetary masses in the case of low-eccentricity orbits.

In Section 2, we begin by describing a harmonic approach to analyzing TTVs. We review the work of Lithwick et al. (2012) in Section 3, and discuss the TTV signal for a system near a first-order mean motion resonance (referred to hereafter as the "Lithwick et al. 2012 formula"). We then introduce the conjunction effect and give analytic formulae for the chopping signal in Section 4; these were derived first in Agol et al. (2005) and more recently, using a similar approach, in Nesvorný \& Vokrouhlický (2014b), though not in the form presented here. We discuss how these formulae depend on orbital parameters and more generally consider the range of validity of the synodic TTV formulae. We then place the synodic TTV expression in context with that of Lithwick et al. (2012) and discuss the regimes in which each should be used. In Section 4.4, we address the more general problem of using these formulae to predict, given the timing precision on the transits, how many observations are required to infer the masses of a particular system. This will be important in the planning of follow-up observations of partially characterized systems and for estimating the timing precision required for future surveys to obtain a measurement of planetary masses through chopping.

In Section 5, we apply the synodic TTV formula to Kepler data, and use it to infer planetary masses for systems both near and far from mean motion resonance. The synodic formulae can be used alone to determine planetary masses, or in combination with the Lithwick et al. (2012) formula for systems near first-order mean motion resonances, in which case the mass-free eccentricity degeneracy can be broken and a constraint on the free eccentricities can be determined as well. We present our conclusions in Section 6. In Appendix A, we present a derivation of the synodic TTV formulae based on Hamiltonian perturbation theory, and we discuss the convergence of the series for the synodic chopping signal in Appendix B.

\footnotetext{
4 Degeneracies have also been identified between eccentricity vector components and between planetary mass and mutual inclination (Carter et al. 2012; Lithwick et al. 2012; Huber et al. 2013).

5 The short-timescale variations occur on harmonics of the synodic timescale, $\left(P_{1}^{-1}-P_{2}^{-1}\right)^{-1}$, but can sometimes appear to vary on a longer timescale due to aliasing (see Section 2).
}

\section{TRANSIT ALIASING AND HARMONIC ANALYSIS OF TTVS}

The TTVs of a planet can be written as a combination of periodic components with frequencies that are integer combinations of the two interacting planets' orbital frequencies, $n_{p q}=p n_{1}-q n_{2}$, where $(p, q)$ are integers, and $n_{1,2}=2 \pi / P_{1,2}$ are the mean motions of the two planets (Nesvorný \& Morbidelli 2008; Nesvorný 2009; Nesvorný \& Beaugé 2010). More explicitly, TTVs for a two-planet system can be expanded as

$$
\begin{aligned}
\delta t_{1, k}= & P_{1} \frac{m_{2}}{M_{\star}} \sum_{p, q}\left[a_{1, p, q} \cos \left[\left(p n_{1}-q n_{2}\right) t_{1, k}\right]\right. \\
& \left.+b_{1, p, q} \sin \left[\left(p n_{1}-q n_{2}\right) t_{1, k}\right]\right], \\
\delta t_{2, k}= & P_{2} \frac{m_{1}}{M_{\star}} \sum_{p, q}\left[a_{2, p, q} \cos \left[\left(p n_{1}-q n_{2}\right) t_{2, k}\right]\right. \\
& \left.+b_{2, p, q} \sin \left[\left(p n_{1}-q n_{2}\right) t_{2, k}\right]\right],
\end{aligned}
$$

where $k$ denotes the transit number, $a_{i, p, q}, b_{i, p, q}(i=1,2)$ are coefficients that are functions of the orbital elements of the planets (except the mean longitudes $\lambda_{i}$ ), and therefore vary on timescales long compared to the orbital period; $m_{1}, m_{2}$ are the masses of the two planets; $M_{\star}$ is the mass of the star; and $t_{i, k}$ is the $k$ th transit time of the $i$ th planet. We assume that the observation baseline is short compared to the secular timescales (which are typically "long" since they are proportional to $\left.P_{i} M_{\star} / m_{\text {planet }}\right)$ so that treating the coefficients $a_{i, p, q}, b_{i, p, q}$ as constant is justified. We will discuss this further in Section 4.2. Note that the TTVs scale in proportion to the orbital period of each transiting planet and in proportion to the mass ratio of the perturbing planet. (These equations and scaling relations do not apply in mean-motion resonance; see Agol et al. 2005.)

The transit times, $t_{i, k}$ are converted to TTVs after removing a mean ephemeris: $\delta t_{i, k}=t_{i, k}-t_{i, 0}-P_{i} k$, where $k$ is an integer (Agol et al. 2005). Since TTVs are typically much smaller than the planetary orbital periods, $\left|\delta t_{i, k}\right| \ll P_{i}$, the planets' transits are (nearly) sampled on their orbital frequencies. In the right hand sides of Equations (2), then, we can replace $t_{i, k}$ with $k P_{i}+t_{i, 0}$ and remain accurate to order $\left(m_{\text {planet }} / M_{\star}\right)$. For example, using this approximation, $p n_{1} t_{1, k}=2 \pi p k+n_{1} p t_{1,0}$, and we can rearrange the expression for the TTVs of the inner planet as follows:

$$
\delta t_{1, k} \approx P_{1} \frac{m_{2}}{M_{\star}} \sum_{q}\left[a_{1, q}^{\prime} \cos \left(q n_{2} t_{1, k}\right)+b_{1, q}^{\prime} \sin \left(q n_{2} t_{1, k}\right)\right]
$$

where e.g., $a_{1, q}^{\prime} \equiv \sum_{p}\left(a_{1, p, q} \cos \left(p n_{1} t_{1,0}\right)+b_{1, p, q} \sin \left(p n_{1} t_{1,0}\right)\right)$. A similar set of steps can be performed for the outer planet, yielding:

$$
\delta t_{2, k} \approx P_{2} \frac{m_{1}}{M_{\star}} \sum_{p}\left[a_{1, p}^{\prime} \cos \left(p n_{1} t_{2, k}\right)+b_{1, p}^{\prime} \sin \left(p n_{1} t_{2, k}\right)\right] .
$$

Therefore, the TTVs of the transiting planet can be written entirely in terms of harmonics of the perturbing planet's orbital frequency. This fact is due to the sampling of the TTV on the transiting planet's orbital period. Terms with frequencies that differ by integer multiples of the orbital frequency of the transiting planet are indistinguishable in the TTVs of that planet. For example, in the expression of the TTVs of the inner planet given in Equation (2), each term with frequency $p n_{1}-q n_{2}$ is 
aliased to the frequency $\mathrm{q} n_{2}$, regardless of the value of $p$. The term "aliasing" refers to the general effect where signals with different intrinsic frequencies are indistinguishable from each other because of discrete sampling. (The aliasing described here is unrelated to the Nyquist limit.)

Note that if the perturbing planet does not transit the host star, this aliasing can mask the true period of the perturbing planet since we can add integer multiples of the transiting planet's orbital frequency without affecting the TTV. If both planets transit the star, however, both periods and orbital phases are known. Consequently, TTVs can be fit using a sum of harmonics of the perturbing planet's orbital frequencyinstead of a double sum of all possible linear combinations of both planet's orbital frequencies-where the only unknowns are the coefficients of these harmonics. This is a linear function of the unknown parameters, and hence can be solved by matrix inversion yielding a unique solution. We refer to this as a "harmonic analysis" and utilize it below to identify components of the TTV that are caused by the conjunctions of the planets (Nesvorný \& Vokrouhlický 2014b), and thus depends on the difference between their mean longitudes, the synodic angle $\psi=\lambda_{1}-\lambda_{2}=\left(n_{1}-n_{2}\right) t+\psi(t=0)$.

It is important to point out that the expression of the TTVs as a sum over harmonics of the perturbing planet's orbital frequency does not mean the TTVs appear with periodicities of integer fractions of $P_{\text {perturber. This unintuitive feature is }}$ because of the discrete times at which these harmonics are sampled. For example, in a system near the 2:1 resonance, the fast frequency $2\left(n_{1}-n_{2}\right)$ will, due to the discrete sampling at every transit of the inner planet, be aliased such that in the TTVs of the inner planet it appears as a sinusoid with the superperiod given in Equation (1), i.e., the same timescale as the resonant frequency $2 n_{2}-n_{1}$. This is akin to the stroboscopic effect in which a spinning car wheel appears to be rotating at a slower rate due to the sampling rate.

\section{FIRST-ORDER RESONANT TTV SIGNAL}

Here we summarize the formulae for the largest amplitude components of TTVs of a pair of planets near a first-order mean motion resonance, as derived by Lithwick et al. (2012), and remind the reader of the origin of the mass-eccentricity degeneracy. These formulae do not apply to a pair of planets which are dynamically in the mean motion resonance (for example with a librating resonant argument).

Lithwick et al. (2012) considered a system of two planets near the $j_{R}: j_{R}-1$ first-order resonance, in which case the TTVs take the following approximate form:

$$
\begin{aligned}
& \delta t_{1}=\Re\left[-i V_{1} \exp i \lambda^{j_{R}}\right] \\
& \delta t_{2}=\mathfrak{R}\left[-i V_{2} \exp i \lambda^{j_{R}}\right]
\end{aligned}
$$

here $i$ denotes $\sqrt{-1}$ and

$$
\begin{aligned}
V_{1} & =\frac{P_{1}}{\pi} \frac{1}{j_{R}^{2 / 3}\left(j_{R}-1\right)^{1 / 3} \Delta} \frac{m_{2}}{M_{\star}}\left[-f-\frac{3 Z_{\text {free }}^{*}}{2 \Delta}\right] \\
V_{2} & =\frac{P_{2}}{\pi} \frac{1}{j_{R} \Delta} \frac{m_{1}}{M_{\star}}\left[-g+\frac{3 Z_{\text {free }}^{*}}{2 \Delta}\right] \\
\lambda^{j_{R}} & =j_{R} \lambda_{2}-\left(j_{R}-1\right) \lambda_{1},
\end{aligned}
$$

with

$$
\begin{aligned}
Z_{\text {free }} & =f e_{1}^{\exp i \varpi_{1}}+g e_{2}^{\exp i \varpi_{2}} \\
\Delta & =\frac{P_{2}}{P_{1}} \frac{j_{R}-1}{j_{R}}-1
\end{aligned}
$$

and $f$ and $g$ are combinations of Laplace coefficients (note that $f<0$ and $g>0), e_{1,2}$ the free eccentricities, and $\varpi_{1,2}$ the longitudes of pericenter of each of the planets. The reference direction from which longitudes are measured is the observer's line of sight, so that $\lambda_{i} \approx 0$ at transit. Note that $V_{1}, V_{2}$, and $Z_{\text {free }}$ are complex quantities; however, all observables are found by taking the real components as in Equation (5). $\left(Z_{\text {free }}^{*}\right.$ is the complex conjugate of $Z_{\text {free }}$, and $\mathfrak{R}(x)$ and $\mathfrak{I}(x)$ denote the real and imaginary components of a complex number $x$ ). In the derivation of these formulae, only the two resonant terms associated with the $j_{R}: j_{R}-1$ resonance at first-order in eccentricity are considered. All other terms in the gravitational potential between the planets, even those of independent of eccentricity, are neglected since near resonance the TTVs they produce are very small compared to those caused by the resonant terms (due to the small denominator $\Delta$ appearing in the amplitudes given in Equations (6)).

The total eccentricity of a planet near a first-order mean motion resonance is made up of "free" and "forced" components; the forced eccentricity is driven by resonant interactions between the planets while the free eccentricity is determined by matching the initial eccentricity vector (the initial conditions). The free eccentricity vector can be approximated as constant on observational timescales (it varies on the long secular timescale) while the forced eccentricity vector precesses on the resonant timescale (the super-period). Both components affect the TTVs: the forced eccentricity is responsible for the first term in the brackets in Equations (6) (depending on the coefficients $f$ and $g$ ), while the free eccentricities are responsible for the $Z_{\text {free }}^{*}$ term. The amplitude of the free eccentricity is not necessarily smaller or larger than that of the forced eccentricity.

In the case of two transiting planets, the unknown quantities are $\mathfrak{R}\left[Z_{\text {free }}\right], \mathfrak{I}\left[Z_{\text {free }}\right], m_{1} / M_{\star}$, and $m_{2} / M_{\star}$, and, in principle, there are four measurable quantities: both amplitudes and both phases. However, as pointed out in Lithwick et al. (2012), in both the case of $\left|Z_{\text {free }}\right| \ll \Delta$ and $\left|Z_{\text {free }}\right| \gg \Delta$ the TTVs are approximately anti-correlated, and so in either of these regimes the relative phase is no longer a constraining quantity (if the signal-to-noise ratio is insufficient to measure a phase offset). This leads to the degeneracy between mass and free eccentricities, and hence from this first-order TTV alone one in practice cannot usually determine the masses or the combination of free eccentricities $Z_{\text {free }}$.

Finally, since the TTVs are sampled for the inner [outer] planet at $\lambda_{1}\left(t=t_{1, k}\right)=\lambda_{1}\left(t=t_{1,0}\right)+2 \pi k$ $\left[\lambda_{2}\left(t=t_{2, k}\right)=\lambda_{2}\left(t=t_{2,0}\right)+2 \pi k\right]$, these expressions become:

$$
\begin{aligned}
& \delta t_{1}=\mathfrak{R}\left[-i V_{1} \exp \left(i j_{R} \lambda_{2}\right)\right] \\
& \delta t_{2}=\mathfrak{R}\left[-i V_{2} \exp \left(i\left(j_{R}-1\right) \lambda_{1}\right)\right] .
\end{aligned}
$$

To be clear, the above equations produce TTVs which are slowly varying with a timescale equal to the super-period of the 
resonance (Equation (1)), and not with timescales of, e.g., $P_{2} / j_{R}$ or $P_{1} /\left(j_{R}-1\right)$. This is because the sampling rate of these periodic functions is nearly resonant with their periodicities, as discussed in Section 2.

Actually, Lithwick et al. (2012) have taken advantage of the aliasing effect to create these expressions. The component of the TTVs due to the forced eccentricity has a $j_{R}\left(\lambda_{2}-\lambda_{1}\right)$ dependence for the inner planet and a $\left(j_{R}-1\right)\left(\lambda_{2}-\lambda_{1}\right)$ dependence of the outer planet (see their Equations (A.15) and (A.24). Because these appear at the same aliased frequency as the resonant $j_{R} \lambda_{2}-\left(j_{R}-1\right) \lambda_{1}$ term, they are included with the resonant TTV. Since the reference direction was chosen such that $\lambda_{i}=0$ at transit, these two terms have the same phase and can be grouped together in this way.

\section{THE SYNODIC CHOPPING SIGNAL}

A pair of planets interacts most strongly when the distance between them is smallest. For low eccentricity and nearly coplanar orbits, this occurs at conjunction, when the synodic angle $\psi=\lambda_{1}-\lambda_{2}=0\left(\lambda_{1}=\lambda_{2}\right)$. Conjunctions occur periodically, with a timescale of $P_{\text {syn }}=2 \pi / n_{\text {syn }}=$ $1 /\left(n_{1}-n_{2}\right)=1 /\left(1 / P_{1}-1 / P_{2}\right)$, and it is intuitive to expect that this timescale would appear in the TTVs. In fact, at zeroth order in the eccentricities, the TTVs only depend on the synodic angle and its harmonics,

$$
\psi_{j}=j \psi=j\left(\lambda_{1}-\lambda_{2}\right) \text {; }
$$

The synodic chopping signal is included in the computations in Agol et al. (2005) and Nesvorný \& Vokrouhlický (2014b). We also give a distinct derivation in Appendix A. Our expression for the TTVs, which can be written entirely as a sum over harmonics of the synodic angle $\psi$, differs slightly from the expressions of Agol et al. (2005) and Nesvorný \& Vokrouhlický (2014b), which include additional constants, terms linear in $\psi$, and sinusoidal terms with frequency $n_{1}$ (in the TTVs of the inner planet) and $n_{2}$ (in the TTVs of the outer planet). We discuss the reason for these extra terms in Appendix A, but note now that these extra terms do not affect the TTVs. Both constants and linear terms in $\psi$ (which are therefore linear in time) are indistinguishable from a slightly different average orbit or mean ephemeris (resulting from a linear fit to the transit times). Moreover, since transits always occur at the same value of $\lambda_{i}$, the extra sinusoidal terms also do not contribute to the TTVs. Therefore, our expressions agree with the previous derivations in the terms that contribute to calculated TTVs (observed transit times minus those expected based on the average orbit), in the limit that the reflex motion of the star can be ignored. Any further differences in notation come from the fact that our expression is given as a sum over harmonics instead of more complicated periodic functions of $\psi$. Note that a published erratum corrects some typos appearing in Nesvorný \& Vokrouhlický (2014b; Nesvorný \& Vokrouhlický 2014a).

\subsection{Synodic Chopping Signal Formulae to Zeroth-order in the Free Eccentricities}

For an inner transiting planet, the synodic component of the TTVs takes the form

$$
\delta t_{1}=\frac{P_{1}}{2 \pi} \frac{m_{2}}{M_{\star}} \sum_{j=1}^{\infty} f_{1}^{(j)}(\alpha) \sin \psi_{j},
$$

where

$$
f_{1}^{(j)}(\alpha)=\alpha \frac{\left(\begin{array}{c}
2 \beta D_{\alpha} b_{1 / 2}^{(j)}(\alpha)+j\left(3+\beta^{2}\right) b_{1 / 2}^{(j)}(\alpha) \\
-\alpha \delta_{j, 1}\left(\beta^{2}+2 \beta+3\right)
\end{array}\right)}{\beta^{2}\left(1-\beta^{2}\right),}
$$

where $\alpha=a_{1} / a_{2}$ is the ratio of semi-major axes, $\beta=j\left(n_{1}-n_{2}\right) / n_{1}, b_{1 / 2}^{(j)}(\alpha)$ is the Laplace coefficient

$$
b_{1 / 2}^{(j)}(\alpha)=\frac{1}{\pi} \int_{0}^{2 \pi} \frac{\cos (j \theta)}{\sqrt{1-2 \alpha \cos \theta+\alpha^{2}}} d \theta
$$

and $D_{\alpha}$ is the derivative operator $\alpha \frac{\partial}{\partial \alpha}$. The Laplace coefficients can be evaluated in terms of complete elliptic integrals; for example:

$$
\begin{aligned}
b_{1 / 2}^{(1)}(\alpha) & =4(K(\alpha)-E(\alpha)) /(\alpha \pi), \\
b_{1 / 2}^{(1)}(\alpha)+\alpha \frac{\partial b_{1 / 2}^{(1)}(\alpha)}{\partial \alpha} & =\frac{4 \alpha E(\alpha)}{\pi\left(1-\alpha^{2}\right)},
\end{aligned}
$$

where $K(k)$ and $E(k)$ are the complete elliptic integrals of the first and second kinds, respectively.

For an outer transiting planet, the synodic component of the TTVs takes the form

$$
\delta t_{2}=\frac{P_{2}}{2 \pi} \frac{m_{1}}{M_{\star}} \sum_{j=1}^{\infty} f_{2}^{(j)}(\alpha) \sin \psi_{j},
$$

where

$$
f_{2}^{(j)}(\alpha)=\frac{\left(\begin{array}{c}
j\left(\kappa^{2}+3\right) b_{1 / 2}^{(j)}(\alpha)+2 \kappa\left(D_{\alpha} b_{1 / 2}^{(j)}(\alpha)\right. \\
\left.+b_{1 / 2}^{(j)}(\alpha)\right)-\alpha^{-2} \delta_{j, 1}\left(\kappa^{2}-2 \kappa+3\right)
\end{array}\right)}{\kappa^{2}\left(\kappa^{2}-1\right)},
$$

where $\kappa=j\left(n_{1}-n_{2}\right) / n_{2}$.

Note that these relations have no eccentricity dependence; they only depend on the semi-major axis ratio of the two planets, $\alpha$, and the planet-star mass ratios. The phase and period of the chopping signal are straightforward to measure if the perturbing planet also transits the star; thus, if this synodic chopping signal can be measured, the mass ratio of the perturbing planet can be immediately inferred if the parameters of the system satisfy the major assumptions made in this paper (see Section 4.2).

In the limit of large $j$, the function $f_{1}^{(j)}(\alpha)$ has a leading coefficient that scales like $\alpha^{j+1} / j$ and the function $f_{2}^{(j)}(\alpha)$ has a leading coefficient that scales like $\alpha^{j+3} / j$ (see Appendix B), so that the largest contributions to the sum in Equation (10) and in Equation (14) in general come from smaller values of $j$. Because of the behavior of the leading coefficients, more terms are necessary to faithfully approximate the TTV signal as $\alpha \rightarrow 1$ because the convergence of the sums is slow.

Closely spaced planets will, at a given $j$, have a larger synodic TTV than more widely spaced planets because the parameters $\beta$ and $\kappa$ appearing in the denominators approach zero as $\alpha \rightarrow 1$. Additionally, if the pair is near the $j_{R}: j_{R}-1$ mean motion resonance, the denominators $\beta^{2}-1$ and $\kappa^{2}-1$ 

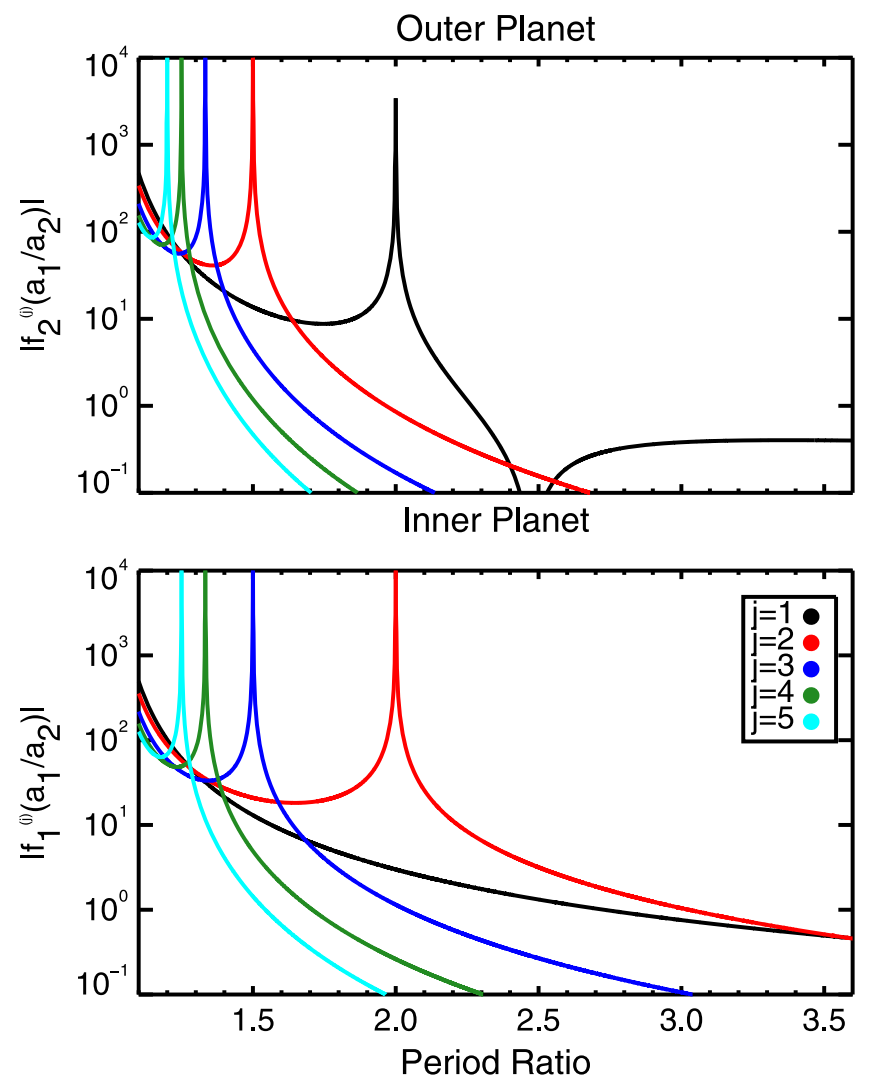

Figure 1. Magnitude of the chopping coefficient functions, $f_{1}^{(j)}(\alpha)$ (bottom) and $f_{2}^{(j)}(\alpha)$ (top), as a function of period ratio, for several values of $j$. Near the $j_{R}: j_{R}-1$ resonance, the inner planet chopping function peaks for $j=j_{R}$, while the outer planet chopping function peaks for $j=j_{R}-1$ (with $j_{R} \geqslant 2$ ).

will be close to zero for the term with $j=j_{R}$ (for the inner planet) and $j=j_{R}-1$ (for the outer planet). Near the $j_{R}: j_{R}-1$ resonant configuration, then, these terms dominate the synodic TTV because of these small denominators. This reflects the fact that near the $j_{R}: j_{R}-1$ mean motion resonance, the time between conjunctions approximately corresponds to $j_{R}-1$ orbits of the outer planet and $j_{R}$ orbits of the inner one, such that most of the TTV amplitude is incorporated in these harmonics of the synodic angle. As discussed in Section 2, these particular harmonics of the synodic angle contribute to the TTV as a long period effect: due to aliasing, they have a timescale given by the super-period of the first-order resonance.

The magnitude of the functions $f_{1}^{(j)}(\alpha)$ and $f_{2}^{(j)}(\alpha)$ are plotted in Figure 1 for $j=1,2,3,4,5$. It is clear that the synodic amplitude $f_{1,2}^{(j)}(\alpha)$ generally grows as $\alpha \rightarrow 1$, for all $j$, and therefore more terms must be included in the sum for close pairs of planets. Furthermore, when the period ratio is close to the $j_{R}: j_{R}-1$ mean motion resonance, the functions with $j=j_{R}$ (inner planet) and $j=j_{R}-1$ (outer planet) peak for the reasons discussed above. This happens for all $j$ at some period ratio except for the $j=1$ synodic TTV of the inner planet since $j_{R} \geqslant 2$. As noted in Agol et al. (2005), the dip near a period ratio of 2.5 in the $j=1$ synodic signal of the outer planet is due to the fact that the TTV caused by the motion of the star about the barycenter of the inner planet-star binary subsystem is opposite in sign and comparable to that caused by direct interaction with the inner planet at this period ratio. For larger period ratios, the TTV of the outer planet is dominated by the component due to the motion of the star about the barycenter of the inner planet-star binary subsystem.

\subsection{Range of Validity of the Synodic Formula}

The intrinsic assumptions made in deriving these TTV expressions were (1) that the system has low eccentricities and nearly coplanar orbits; (2) that the system is not in or too near resonance; (3) that $\alpha$ is not too close to unity; (4) that the masses of the planets are small compared to that of the star; and (5) that the coefficients themselves can be treated as constant in time. Additionally, if two perturbing planets contribute to the TTVs of a third planet, the true TTV of this third planet cannot be written as only a sum of one of the perturbing planet's harmonics. In most configurations, we hypothesize that the TTV could be approximated as a simple sum of two "singleperturber" contributions.

First, we consider the qualification of "small" eccentricities and inclinations. Neglected terms linear in eccentricity or inclinations would lead to an error in the coefficients of $O(e)$ and $O(i)$. Therefore, if the eccentricities and inclinations are only a few percent than the error will also be a few percent. However, whether or not the eccentricities and inclinations can be considered "small" depends on how close the system is to resonance.

In general, if one derived a formula for the TTV good to first-order in eccentricity, it would have terms at first-order in eccentricity only depending on the angles of $j \lambda_{2}-(j-1) \lambda_{1}$ (not including the longitude of pericenter piece). In fact, the TTV formulae themselves should have the d'Alembert characteristics since they only depend on angles referenced to a fixed direction (longitudes) (Hamilton 1994). Due to the transit aliasing effect discussed above, these first-order frequencies will be indistinguishable from synodic-type variations of frequency $j\left(n_{1}-n_{2}\right)$ for the inner planet since these appear as the same harmonic of the outer planet, $j n_{2}$ (see Equation (8)). For the outer planet, these first-order (in eccentricity) terms will be indistinguishable from those at the harmonic of $(j-1)\left(n_{1}-n_{2}\right)$.

These first-order terms will have amplitude proportional to $e /\left(j n_{2}-(j-1) n_{1}\right)$, which, away from resonance, should be negligible for all $j$ if the eccentricity is low. But near the $j_{R}$ : $j_{R}-1$ resonance, the $j_{R} n_{2}$ (for the inner planet) and the $\left(j_{R}-1\right) n_{1}$ term (for the outer planet) will have amplitudes proportional to $e /\left(j_{R} n_{2}-\left(j_{R}-1\right) n_{1}\right)$ (appearing as $e / \Delta$ in the formulae of Lithwick et al., Equation (6)). The resonant combination of frequencies in the denominator mitigates the effects of the eccentricity coefficient, so that these terms are large corrections to the TTV formulae at these frequencies, and hence they make the synodic formula for these values of $j$ less accurate. Note that since $j_{R} \geqslant 2$, the synodic frequency, $n_{1}-n_{2}$, has no resonant aliases for the inner planet. Thus, the harmonic component, which depends on $n_{2}$, can be uniquely identified with the synodic frequency variation.

If the system is in a mean motion resonance, the dominant, resonant TTV period will be related to the libration time. In this case, the resonant contribution to the TTVs will not appear at the frequency $j_{R} n_{2}$ for the inner planet and $\left(j_{R}-1\right) n_{2}$ for the outer planet, since the libration time cannot be written simply as an integer times the orbital frequency. In the resonant case, then, the harmonic analysis approach cannot be used. 

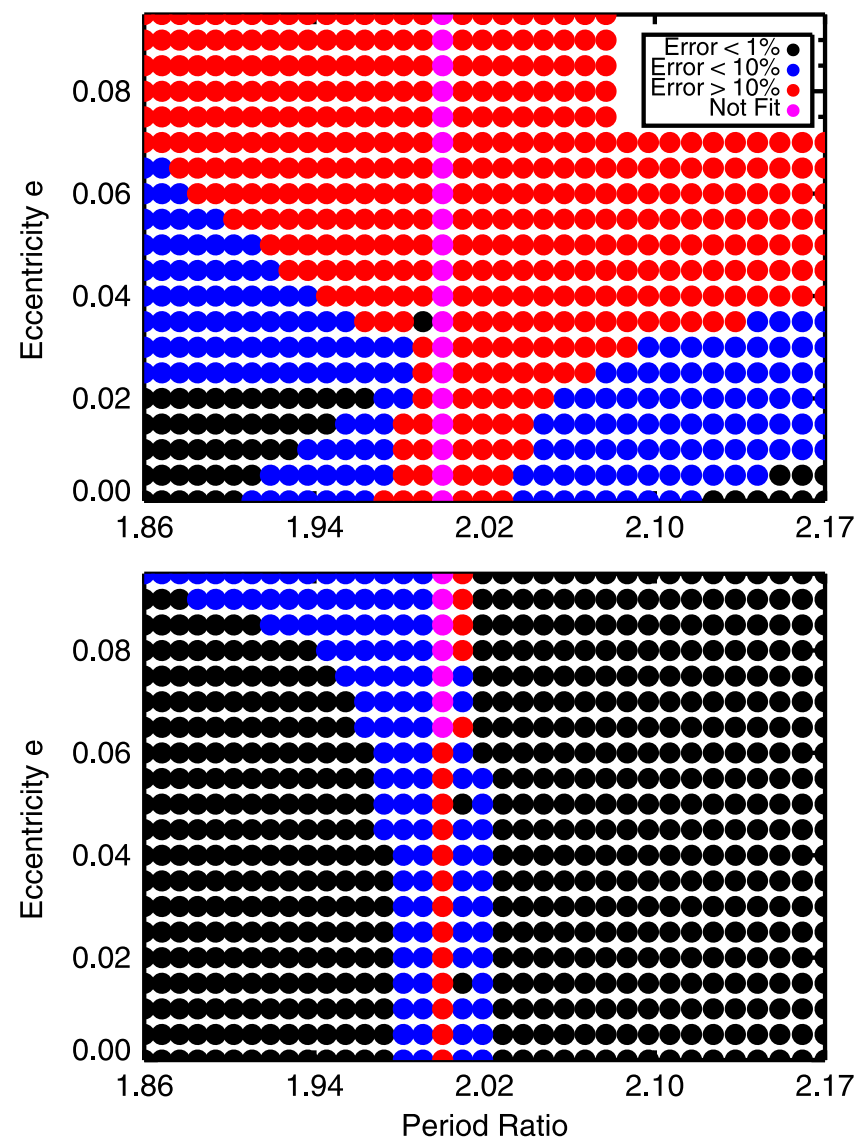

Figure 2. Comparison of the synodic formula with $j=1$ with the measured harmonic with frequency $n_{2}$ of simulated TTVs for the inner planet (bottom), and with frequency $n_{1}$ of simulated TTVs for the outer planet (top). The color scale denotes the fractional error in the chopping formula as a function of the period ratio of the pair and the eccentricities of the orbits, where the vertical scale is $e=\left(e_{1}^{2}+e_{2}^{2}\right)^{1 / 2}$. Black points denote errors $E<1 \%$, blue those with errors $1 \%<E<10 \%$, and red those with errors $E>10 \%$. We did not fit the TTVs of the systems in magenta, because the fitting method failed (because the TTV period was significantly unresolved by the simulation time). For the outer planet we purposely picked the harmonic $j=1$, which is aliased to the resonant frequency in order to show how higher order eccentricity effects are important for understanding the resonant term.

It is important to point out that in each of these cases-both near the $j_{R}: j_{R}-1$ resonance, where eccentricity errors are larger, and in the mean motion resonance, where the derivation in Appendix A must be modified, the synodic TTV formula for values of $j \neq j_{R}$ (inner planet) and $j \neq j_{R}-1$ (outer planet) will still apply.

In Figure 2, we show how the predicted amplitude of the $j=1$ synodic chopping term compares with that calculated numerically, for both the inner and outer planet, assuming the pair is near the 2:1 resonance. We varied only the period of the outer planet and the eccentricity of both planets (assumed to be equal; the vertical scale is the square root of the sum of the squares of the eccentricities, $\left.e=\left(e_{1}^{2}+e_{2}^{2}\right)^{1 / 2}\right)$. The mass of each planet was set to be $10^{-5} M_{\star}$. For each planet, we first determined the TTVs by fitting a line to the transit times, and we then used the computed average orbital periods to determine the value of $\alpha$ used in the synodic formulae with $j=1$ for each planet. We then determined the numerical amplitude of the chopping signal by fitting 10 harmonics of the perturber's period along with a linear term to 1000 simulated TTVs of the inner planet and between 460 and 540 simulated TTVs of the outer planet and selecting the $j=1$ harmonic. This experiment therefore represents an ideal case where the main source of error comes from the assumptions made in deriving the formulae, and not from issues with not having enough data and/ or precision to resolve the amplitudes of the various harmonics.

The error in the formula for the inner planet is below $1 \%$ across the entire range studied, except very near the 2:1 resonance itself. This is as expected-near the 2:1 resonance, only the $j=2$ contribution of the TTV of the inner planet is expected to have large corrections due to eccentricity effects. The configurations with errors of $>10 \%$ are likely those in the mean motion resonance, where the harmonic approach does not apply (the libration frequency is not simply aliased with the frequency $2 n_{2}$ ), or those where the super period is significantly longer than the simulation time (to be discussed at the end of this section). For the outer planet, the $j=1$ synodic term is aliased with the $j_{R}=2$ resonant term, and hence we expect large errors as a function of eccentricity. Indeed, even relatively far from resonance the synodic formula with $j=1$ fails (errors larger than $10 \%$ ) for eccentricities larger than 0.04 .

We performed the same numerical experiment varying the number of harmonics fit to the TTVs, and we also decreased the simulation time. We found the same results even with fewer harmonics (in all cases, the number of data points was much larger than the number of free parameters). With shorter simulation times, we also recovered the same results except when the simulation time became significantly shorter than the TTV period (in which case the error is not due to the formula, but due to insufficient coverage).

Although we have not calculated the effects of inclination on the TTVs, if the TTVs in fact follow the d'Alembert characteristics then the correction due to inclination will only appear at second order. Therefore, one would expect that the inclination terms neglected will be a smaller source of error than the eccentricity terms neglected. Indeed, Nesvorný \& Vokrouhlický (2014b) did not find errors larger than 20\% in the synodic chopping formula (away from resonance) until the mutual inclination was larger than $50^{\circ}$.

In deriving the TTV formula, we began from the disturbing function, which assumes that the interaction between the two planets can be written as a converging series in $\alpha$. This means that the formulae will not work for co-orbital planets (see Vokrouhlický \& Nesvorný (2014) for an analysis of that case). Additionally, as $\alpha \rightarrow 1$, the Laplace coefficents converge less quickly, and so higher order eccentricity and inclination terms, ignored in the derivation, are potentially more important. A different issue is that as $\alpha \rightarrow 1$, mean motion resonances are densely spaced (for arbitrary $\alpha$, the system is more likely to be close to a resonance if $\alpha$ is closer to unity). In principle then the (neglected) effects of eccentricity could be more important for closer pairs of planets due to the effect of the small denominators discussed above. Note, however, again the special case of the $j=1$ synodic TTV of the inner planet. At $j=1$ there are no possible small denominators in the TTV of the inner planet, since all resonances (except the 1:1) require $j \geqslant 2$.

In Figure 3, we show the error in the formula for the $j=1$ synodic TTV, for the inner planet, across a wide range of orbital separation and eccentricities. In making this plot, 500 transits of the inner planet were simulated and 10 harmonics were fit to the resulting TTVs. Except at resonance, the error is less than $10 \%$, regardless of the eccentricity. This indicates that the neglected eccentricity terms are in fact small across a wide 


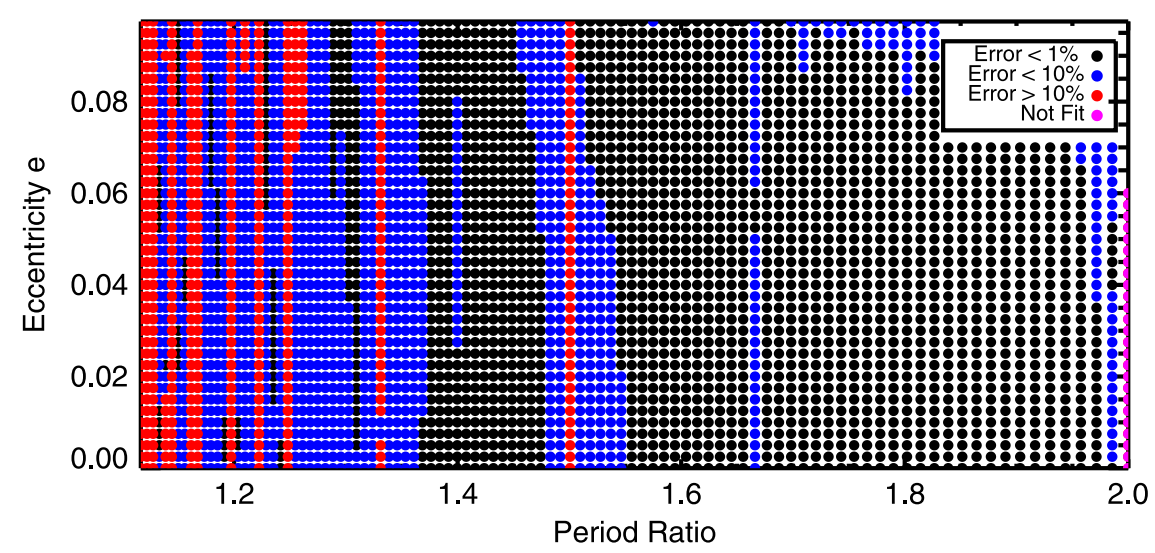

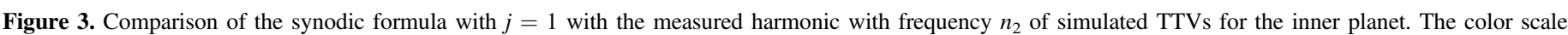

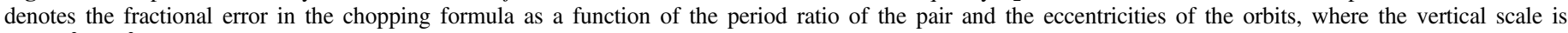

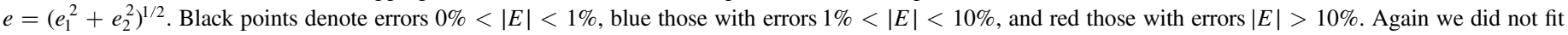
the TTVs of the systems in magenta.

range of $\alpha$. Note that the error is unbiased, in that it is not typically positive or negative.

In deriving the synodic formulae, we assumed that the masses of the planets, compared to that of the host star, are small, so that neglected corrections of the order of $\left(m_{\text {planet }} / M_{\star}\right)^{2}$ are small. In Figure 4, we show how the fractional error in the synodic TTV formula with $j=1$ for the inner planet grows as we increase the masses of the planets to $10^{-4} M_{\star}$ and then to $10^{-3} M_{\star}$. Some of these configurations tested were unstable, and some were perturbed enough to change the number of transits by more than 1 , and those were not fit (though shown in magenta). Note that the widths of resonances grow as the mass of the planets grow, and so more systems are in resonance, where the harmonic analysis will not work, than in the fiducial $m_{\text {planet }} / M_{\star}=10^{-5}$ case shown in Figure 3 . In the case of Jupiter mass planets, there are configurations where the chopping formula may be too approximate, as even outside of resonance, between the $3: 2$ and the $2: 1$, the error is on the order of $30 \%$. As Nesvorný \& Vokrouhlický (2014b) point out, in these cases the chopping formula primarily provides motivation and understanding as to how mass measurements from TTVs arise.

Last, we assume that the coefficients of the synodic TTV, which depend on $\alpha$, can be treated as fixed in time at their average values. It is possible, especially in the near resonant case where the TTV timescale is long, that the observations will cover only a small fraction of the TTV cycle. In this case, the observed semi-major axis ratio may be different than the average one. ${ }^{6}$ The fractional error resulting from using the "incorrect" value of $\alpha$ in the formula for $f_{i}^{(j)}(\alpha)$ will be of order $\left[1 / f_{i}^{(j)}(\alpha)\right]\left[\partial f_{i}^{(j)}(\alpha) / \partial \alpha\right] \delta \alpha$. Although $\delta \alpha$ is small, of the same order of the TTV compared to the orbital period, the derivative of the coefficient $f_{i}^{(j)}(\alpha)$ can be large near resonance. Therefore, if one observes only a small fraction of the super-period of a near resonant system, there will be errors relating to an incorrect estimate of the average value of $\alpha$. These errors are larger for the terms in the synodic sum aliased with the resonant frequency.

\footnotetext{
6 Note also that what we really measure from transit times are average periods. There is a small difference between the true average semi-major axes and those you would estimate from the average periods, which grows for larger mass planets and more closely spaced orbits (Ferraz-Mello et al. 2005).
}

The long-period oscillations in the eccentricity and inclinations due to secular effects will not be resolved, since the secular timescale is in general very long compared to observational timescales (for example, on secular timescales $Z_{\text {free }}$ will change). The synodic chopping formula, which is independent of eccentricity and inclination, will therefore have slowly varying error terms, but as long as the eccentricities and inclination remain small over the secular timescale the error terms will remain small as well.

\subsection{Comparison to the Lithwick et al. Formula with $Z_{\text {free }}=0$}

As discussed at some length in Section 2, the TTVs caused by synodic effects can be aliased such that they are indistinguishable from the resonant, long-period TTV for system near resonance. Not all of these synodic terms were considered in the derivation of the Lithwick et al. (2012) formulae.

Although the Lithwick et al. (2012) formulae contains the dominant contribution at linear order in eccentricity, we can compare the two formulae in the regime where $Z_{\text {free }}=0$, to see how large of an impact the neglected synodic terms have. We expect that near resonance these neglected terms are small, but that the error incurred by neglecting them grows with the distance from resonance. Our expectation is borne out by a numerical comparison between the two, the results of which are shown in Figure 5. In short, the Lithwick et al. (2012) expression is a good approximation to the TTV of systems near first-order mean motion resonances, while further away from resonance it becomes a worse approximation to the chopping signal with $j=j_{R}$ for the inner planet and $j=j_{R}-1$ for the outer planet. Of course, for systems closer to resonance the synodic formulae is a worse approximation at these specific values of $j$ because of eccentricity effects, as we neglect the $Z_{\text {free }} \neq 0$ correction-see Section 4.2. And neither formula for the TTV at the resonant harmonic will apply for a system in a mean motion resonance.

\subsection{Measurement Precision}

One can use the synodic TTV formulae to estimate the precision on the mass measurement of a perturbing planet. For example, the expected mass precision (of the outer planet) due 


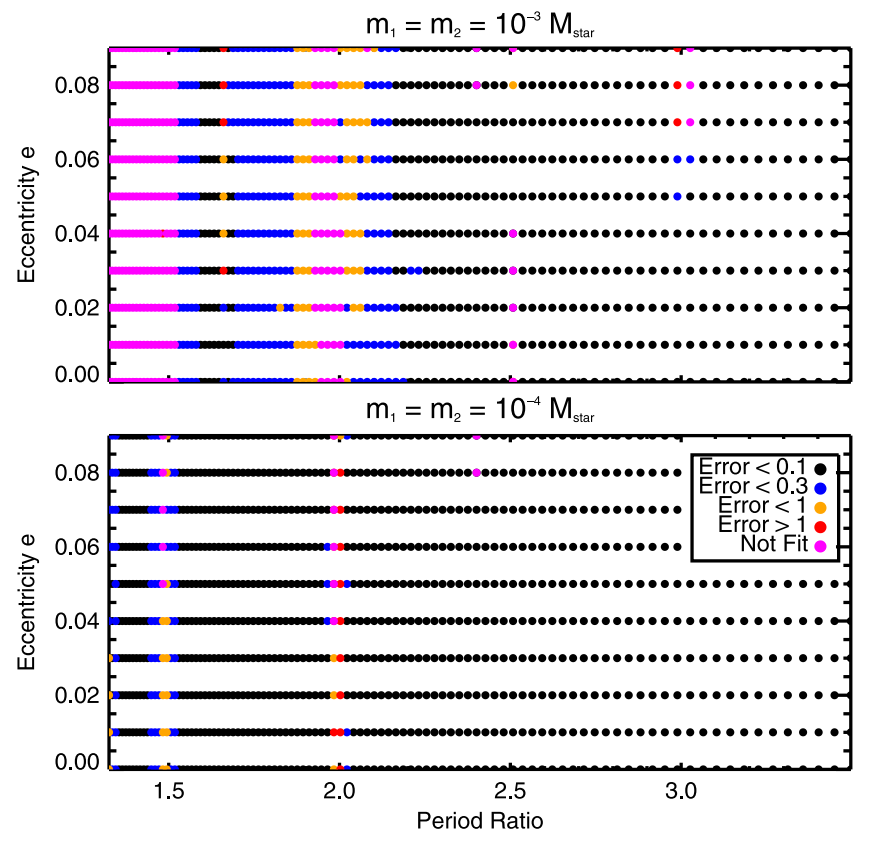

Figure 4. Comparison of the synodic formula with $j=1$ with the measured harmonic with frequency $n_{2}$ of simulated TTVs for the inner planet for planets of mass $10^{-4} M_{\star}$ (bottom) or $10^{-3} M_{\star}$ (top). The color scale denotes the fractional error in the chopping formula as a function of the period ratio of the pair and the eccentricities of the orbits, where the vertical scale is $e=\left(e_{1}^{2}+e_{2}^{2}\right)^{1 / 2}$. Points in magenta had strongly perturbed orbits, with the number of transits varying by at least a few from the expected value, or else had TTV period significantly longer than the simulation time, and were not fit.

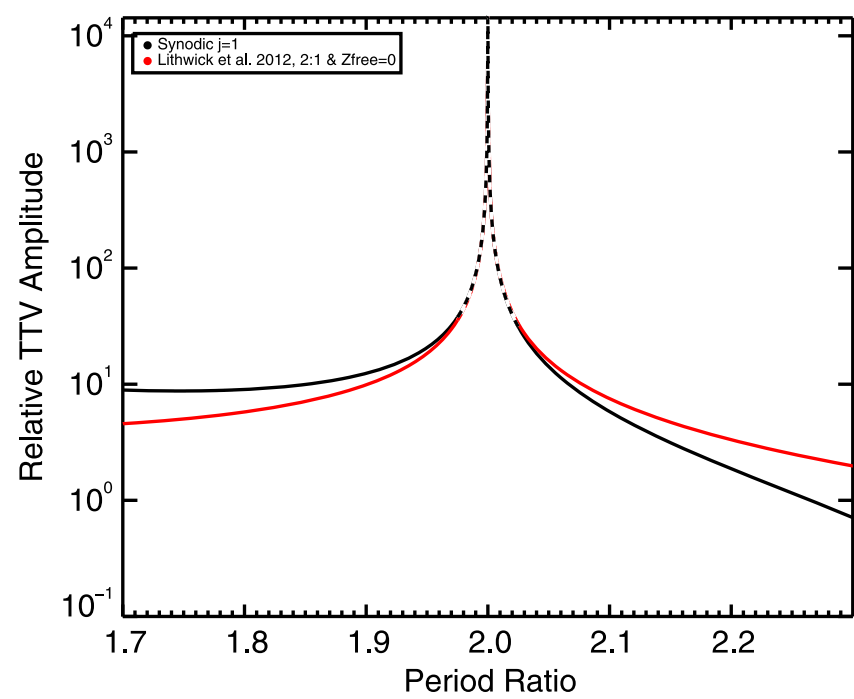

Figure 5. Comparison of the amplitude of the synodic TTV of the outer planet, with $j=1$, and the amplitude from the TTV formula of Lithwick et al. 2012 with $Z_{\text {free }}=0$. Both the close agreement near the resonant period ratio of 2:1 and the disagreement further away is expected. There is a $\sim 25 \%$ error in the Lithwick et al. (2012) formula at a period ratio of 2.1 or 1.9. The dashed line indicates that neither formula applies for systems in a resonance (where we have calculated the width of the resonance at zero eccentricity for planets of equal mass $m_{\text {planet }} / M_{\star}=10^{-5}$ ).

to synodic chopping with $j=1$ in the inner planet is given by

$$
\sigma_{m_{2}}=\sigma_{t_{1}} M_{\star} \frac{2 \pi}{\sqrt{\left(N_{\text {trans }}-N_{\text {param }}\right) / 2} P_{1} f_{1}^{1}(\alpha)},
$$

where $\sigma_{t_{1}}$ is the timing precision of the inner planet, $N_{\text {param }}$ is the number of model parameters, and $N_{\text {trans }}$ is the total number of transits observed. This formula assumes that the phase of the sine function is adequately sampled so that the rms of $1 / \sqrt{2}$ can be assumed, that there is no uncertainty on the mass of the star, $P_{1}$, or $\alpha$, and that there is no covariance with other harmonics being fit. This formula also assumes that transits are observed continuously over the full super-period.

\section{APPLICATIONS}

In this section we apply the chopping and resonant formulae to several planetary systems which have been analyzed using a full dynamical model involving numerical integration of the gravitational equations of motion. We instead analyze these systems using the synodic formulae for TTVs, in combination with the $Z_{\text {free }} \neq 0$ component of the Lithwick et al. (2012) formula for near resonant systems (we use our own expression for the TTV independent of eccentricity, in light of the discussion of Section 4.3). As a result, we can measure planetary masses for these systems without full numerical analysis and without the complication of the mass-free eccentricity degeneracy inherent in the Lithwick et al. (2012) formula alone. This allows us to demonstrate empirically the validity of the synodic TTV signal and to strengthen the understanding of what information in a TTV signal leads to mass measurements.

There are two recipes which one can follow in order to make use of these formulae.

(1) Linear harmonic fitting, followed by translation of the coefficients into mass-ratios. The steps are

(a) carry out a linear fit of the transit times to get an initial estimate of $t_{0, i}, P_{i},(i=1,2)$. Use the mean values of $P_{i}$ to estimate $\alpha$.

(b) Then for each planet, carry out a linear fit:

$$
\begin{aligned}
t_{n, 1}= & t_{0,1}+n P_{1}+\sum_{j=1}^{j_{\max }} a_{j} \sin \left(j 2 \pi\left(t_{0,1}+n P_{1}-t_{0,2}\right) / P_{2}\right) \\
& +b_{j} \cos \left(j 2 \pi\left(t_{0,1}+n P_{1}-t_{0,2}\right) / P_{2}\right),
\end{aligned}
$$

(and a similar equation for planet 2), keeping the ephemerides (the values of $P_{i}$ and $t_{0, i}$ ) of the companion planet fixed at the value from step (a). This can be iterated if needed. The user can determine how many values of $j$ are necessary to give a good fit based on the particular system, but if the system is near a mean motion resonance the Lithwick et al. (2012) $Z_{\text {free }} \neq 0$ formula should be used for coefficient of the $j_{R}\left(\lambda_{1}-\lambda_{2}\right)$ harmonic in the TTVs of the inner planet and the $\left(j_{R}-1\right)\left(\lambda_{1}-\lambda_{2}\right)$ harmonic in the TTVs of the outer planet. Note again that the resonant term and the synodic terms aliased with the resonant term will in general have different phases, unless the reference direction is chosen so that $\lambda=0$ at transit. Additionally, while only the sinusoidal component of the $j\left(\lambda_{1}-\lambda_{2}\right)$ harmonics should have nonzero amplitude since we have accounted for the synodic phase in Equation (17), the resonant component $j_{R} \lambda_{2}-\left(j_{R}-1\right) \lambda_{1}$ has in general both sine and cosine components due to the complex quantity $Z_{\text {free }}$.

(c) use the analytic expressions for $a_{j}$ to compute masses for the coefficients that are non-resonant, and the 
(modified) Lithwick et al. (2012) formula to constrain mass/free eccentricity.

(d) check that the $b_{j}$ values are small for the harmonics which only contain contributions from the synodic terms.

(2) Nonlinear model lightcurve fitting. This involves

(a) computing the coefficients $a_{j} \& b_{j}$ from the model parameters: $\left(t_{0, i}, P_{i}, m_{i} / M_{\star}, e_{i}, \omega_{i}\right)$ for planets $i=1,2$ (and allowing some to float if their values are not well represented by our model, but they contribute significantly to the TTVs);

(b) using a nonlinear solver (or Markov chain) to simultaneously solve for the parameters and uncertainties of both planets. This should give a selfconsistent solution. The former has the advantage that it guarantees a global solution, it is easy to implement, and each planet can be treated individually. Also, error propagation can be carried out linearly. The disadvantage is that the coefficients are not forced to correspond to physical values of the parameters. The latter has the advantage that it gives a self-consistent solution for both planets (although it could still be unphysical if the unconstrained coefficients have amplitudes that are not consistent with the system parameters).

In practice we start with the first technique, see if the results look reasonable, and then use them to initalize the second method, using a Markov chain, to derive the uncertainties, as described below.

\subsection{PH3/Kepler -289}

The Kepler-289 (PH3/KOI-1353/KIC 7303287) planetary system was identified by the Kepler pipeline (Borucki et al. 2011; Batalha et al. 2013; Tenenbaum et al. 2013) and by the Planet Hunters crowd-sourced project (Fischer et al. 2012). This system consists of three planets with orbital periods near 35 days (Kepler-289b/PH3b), 66 days (PH3c), and 126 days (PH3d); each adjacent pair of planets is close to a period ratio of 1:1.9 (Schmitt et al. 2014). The outer two planets both display large amplitude transit-timing variations with a timescale of the super-period of the nearby 2:1 resonance, and the middle planet shows a strong chopping signal caused by the outermost planet. The masses of the outer two planets were measured by the TTVs through a full numerical analysis of the (assumed coplanar) system, performed by EA, as part of Schmitt et al. (2014). The inner planet does not have significantly detected TTVs, and does not significantly affect the outer two planets' transit times, resulting in an upper limit on its mass only.

In this work, we returned to the published transit times and uncertainties of Schmitt et al. (2014) and analyzed them using the harmonic-fitting approach described in Section 2 in order to measure the masses of $\mathrm{PH} 3 \mathrm{c}$ and $\mathrm{PH} 3 \mathrm{~d}$. Figure 6 shows our initial harmonic fit to the data; two harmonics are required for $\mathrm{PH} 3 \mathrm{c}$, while only one is required for $3 \mathrm{~d}$. We ignore the innermost planet (PH3b) in the analysis. We used the $Z_{\text {free }} \neq 0$ component of the Lithwick et al. (2012) formula with $j_{R}=2$ for modeling the $n_{1}$ component of the outer planet's TTVs and for the $2 n_{2}$ component of the middle planet's TTVs. We included the synodic chopping signal with $j=1,2$ for the middle planet PH3c, and the $j=1$ component for the outer

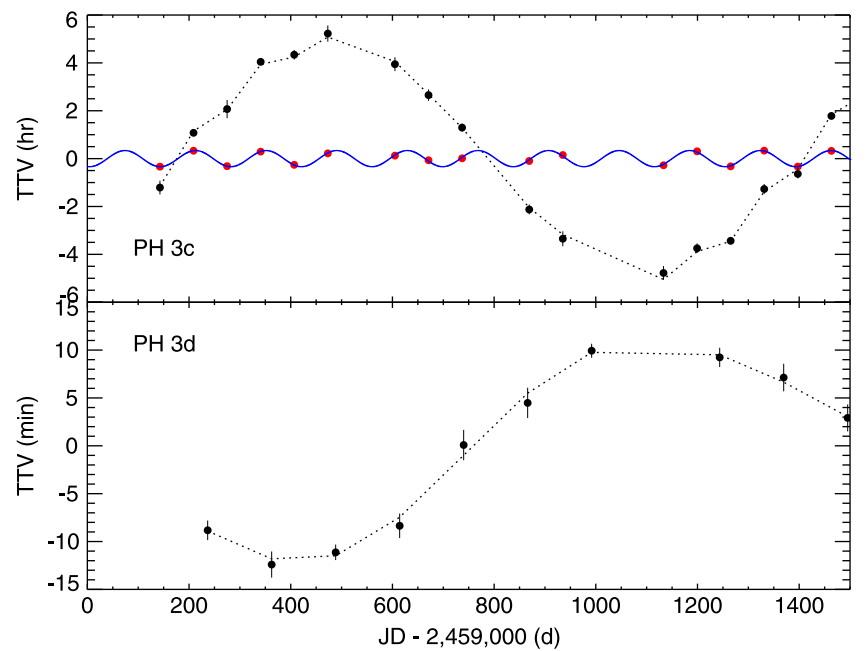

Figure 6. Results of a harmonic analysis for PH $3 \mathrm{c}$ (top) and $3 \mathrm{~d}$ (bottom). In black circles we show the full TTVs for each planet with the measurement uncertainties; note that the top panel is in units of hours, while the bottom is in units of minutes. The dotted curves are the best fit to the TTV using a harmonic analysis with two (one) harmonics of the perturbing planet's orbital frequency for $\mathrm{PH} 3 \mathrm{c}(\mathrm{d})$. In red is the measured chopping $(j=1)$ component for $\mathrm{PH} 3 \mathrm{c}$. The blue solid curve shows the predicted chopping signal based on the Schmitt et al. (2014) mass ratio for the outer planet.

planet (which encompasses the $Z_{\text {free }}=0$ contribution from the Lithwick et al. 2012 formula).

As these three terms together only constrain a linear combination of the free eccentricities of the planets $\left(Z_{\text {free }}\right)$, we also enforced as a prior the Hill stability criterion (Gladman 1993) to prevent the eccentricities from growing too large. We added a systematic error parameter, $\sigma_{0}$, in quadrature to the measured timing errors, such that larger values of $\sigma_{0}$ are penalized in the likelihood function while smaller values of $\sigma_{0}$ require a closer fit to the transit times in order to have a high likelihood. We carried out an affineinvariant Markov chain analysis (Foreman-Mackey et al. 2013) with eleven free parameters: the ephemerides $\left(t_{0}, P\right)$, eccentricity vectors $(e \cos \omega, e \sin \omega)$, and masses of each planet, plus $\sigma_{0}$.

Figure 7 shows the confidence limits in the planet masses from the harmonic analysis with and without the constraint from the synodic chopping signal, as well as the confidence limits from the full dynamical analysis of Schmitt et al. (2014). As explained in Lithwick et al. (2012), the 2:1 resonant signal constrains a combination of the mass ratios of the planets and the free eccentricity, $Z_{\text {free }}$. Without the synodic chopping signal, our analysis shows a banana-like degeneracy between the two planet masses which is due to the trade-off between their masses and the free eccentricity (Figure 7, red), giving $M_{c}=12 \pm 10 M_{\oplus}$ and $M_{d}=219 \pm 57 M_{\oplus}$. When the $j=1$ chopping signal in the TTVs of the middle planet is included, the mass ratio of the outer planet becomes constrained; this then breaks the mass/free eccentricity degeneracy, and allows the mass of the inner planet to be determined as well. The derived error ellipse is similar to that from the full dynamical analysis: Schmitt et al. (2014) report masses of $M_{c}=4.0 \pm 0.9 M_{\oplus}$ and $M_{d}=132 \pm 17 M_{\oplus}$, while the harmonic analysis yields $M_{c}=4.3 \pm 1.1 M_{\oplus} \quad$ and $M_{d}=140 \pm 17 M_{\oplus}$. In comparison, the mass measurements 


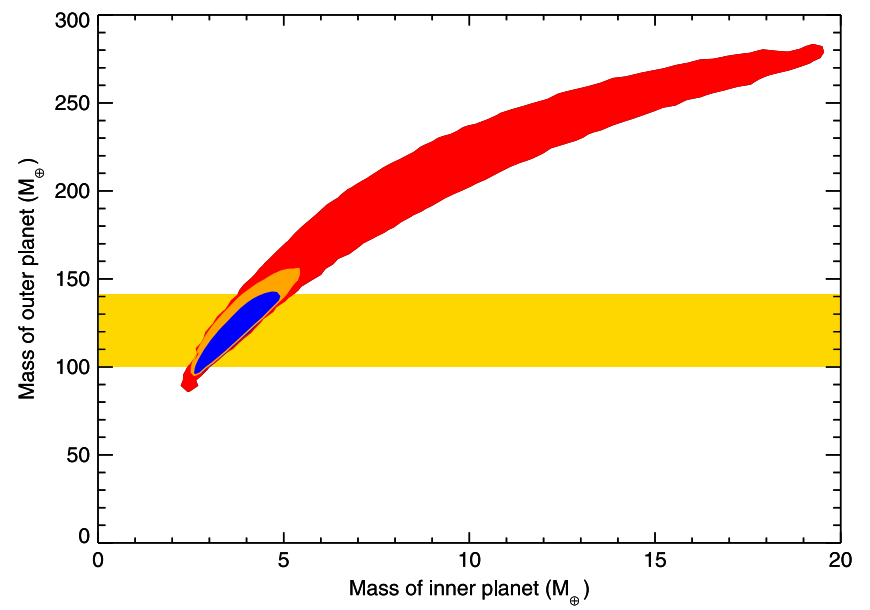

Figure 7. Derived 1- $\sigma$ confidence limits for $\mathrm{PH} 3 \mathrm{c}$ and $\mathrm{d}$. The red is derived from the harmonic analysis without fitting the synodic chopping signal. The orange confidence limit includes synodic chopping, while the blue shows the results from the full dynamical analysis in Schmitt et al. (2014). The constraint on the mass of the outer planet from the chopping signal alone is marked by the yellow horizontal region.

are $M_{c}=12 \pm 10 M_{\oplus}$ and $M_{d}=219 \pm 57 M_{\oplus}$ when the synodic chopping is not included.

This analysis demonstrates the power of the chopping signal in constraining planetary masses near a first-order mean motion resonance. In principle, although the $j \geqslant 2$ chopping components (for the outer planet) and $j \geqslant 3$ chopping components also provide independent constraints on the planetary masses, they are smaller in amplitude and not detected in this case.

\subsection{Kepler $11 \mathrm{~d} / \mathrm{e}$}

The Kepler-11 system (Lissauer et al. 2011) is a system with six transiting planets. A full dynamical analysis has been carried out for this system, giving constraints on the masses of all planets (Lissauer et al. 2013; Migaszewski et al. 2013). Several of the planets, despite being only a few Earth masses, have low densities which require $\mathrm{H} / \mathrm{He}$ atmospheres; this result is puzzling in light of core-accretion theory, which would not predict planets so low in mass to accumulate substantial gaseous envelopes. Here we validate the existing mass measurements for two of these planets and we show that the mass constraints of Kepler-11d and Kepler 11-e largely result from the chopping TTV signal.

Kepler $11 \mathrm{~d}$ and $11 \mathrm{e}$ are two of the three most massive planets in the Kepler -11 system, with periods near 23 and 32 days, respectively, in close proximity to $3: 2$ commensurability. Each of these planets have TTVs that are dominated by the other; thus they can be dynamically "decoupled" from the rest of the planets, and treated as a two-planet system. Note, however, that the decoupling is "one-way": Kepler 11e affects the TTVs of planet Kepler 11f, and hence there is more information with regards to the masses of $\mathrm{d}$ and e to be gained by fitting the entire system instead of treating the $(\mathrm{d}, \mathrm{e})$ pair in isolation.

Figure 8 shows the harmonic fitting results for Kepler $11 \mathrm{~d} /$ e using the transit times due to Jason Rowe presented in Lissauer et al. (2013), to be compared to the dynamical constraints in Table 7 of that paper. In black circles, we show the actual TTV measurements for each planet, with corresponding uncertainties. In this case we simply fit for the

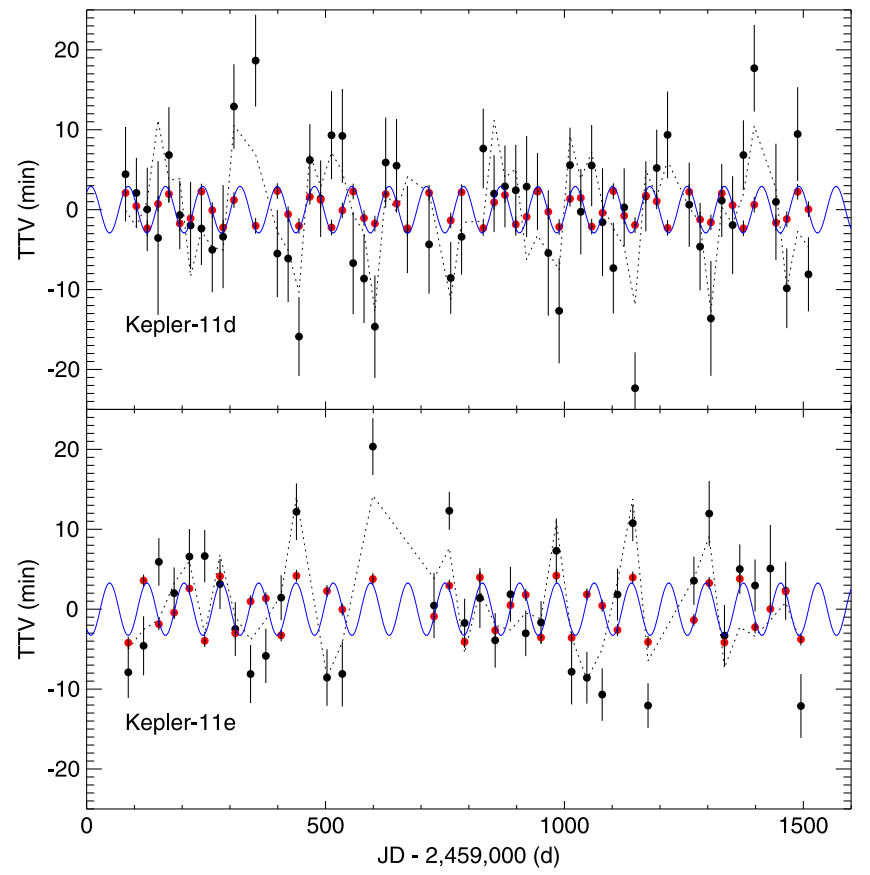

Figure 8. Results of a harmonic analysis for Kepler 11d (top) and 11e (bottom). In black circles we show the full TTVs for each planet with the measurement uncertainties. The dotted curves are the best fit to the TTV using a harmonic analysis with three harmonics of the perturbing planet's orbital frequency. In red is the predicted chopping $(j=1)$ signal for each planet. The blue solid curve shows the predicted chopping signal based on the Lissauer et al. (2013) results.

harmonics of the TTV with the frequency of the companion planet up to $j=3$ (dotted lines), which resulted in excellent $\chi^{2}$ fits for both; the $j=1$ synodic chopping signal from the harmonic fit is also plotted (in red). Note that near the 3:2 mean motion resonance, the $j=1$ synodic signal is not aliased with the resonant frequencies, and so we expect the chopping signal to be well approximated by our formula, as discussed in 4.2. We over plot the predicted $j=1$ synodic chopping signal based on the the best-fit mass ratios from Table 7 in Lissauer et al. (2013), shown as the blue curves. This shows that the chopping signal is detected for both planets, and that it is consistent with the chopping signal predicted by the full dynamical analysis.

Next, we carried out a Markov chain analysis for these planets including the $Z_{\text {free }} \neq 0$ Lithwick et al. (2012) resonance formulae with $j_{R}=3$, relevant to the $3: 2$ commensurability (Section 3 ), as well as both the inner and outer chopping formulae, summed to $j=4$. Figure 9 shows the constraints on the masses of the two planets. The black curve shows the $1 \sigma$ confidence limit from dynamical analysis in Lissauer et al. (2013). In dark[light] blue is the $1[2] \sigma$ confidence limit for our analysis with the resonant and full chopping signals included for both planets; this is consistent with the dynamical analysis at the $<2 \sigma$ level, albeit with a larger uncertainty (recall again that the TTVs of planet Kepler $11 \mathrm{f}$ are affected by Kepler 11-e, and so there is more information as to the $(\mathrm{d}, \mathrm{e})$ subsystem, available when fitting the whole system).

Another interesting byproduct of the chopping signal, when used in conjunction with the Lithwick et al. (2012) formula, is that it allows for a measurement of the quantity $Z_{\text {free }}$. In the case of Kepler $11 \mathrm{~d} / \mathrm{e}$, we find a value of 


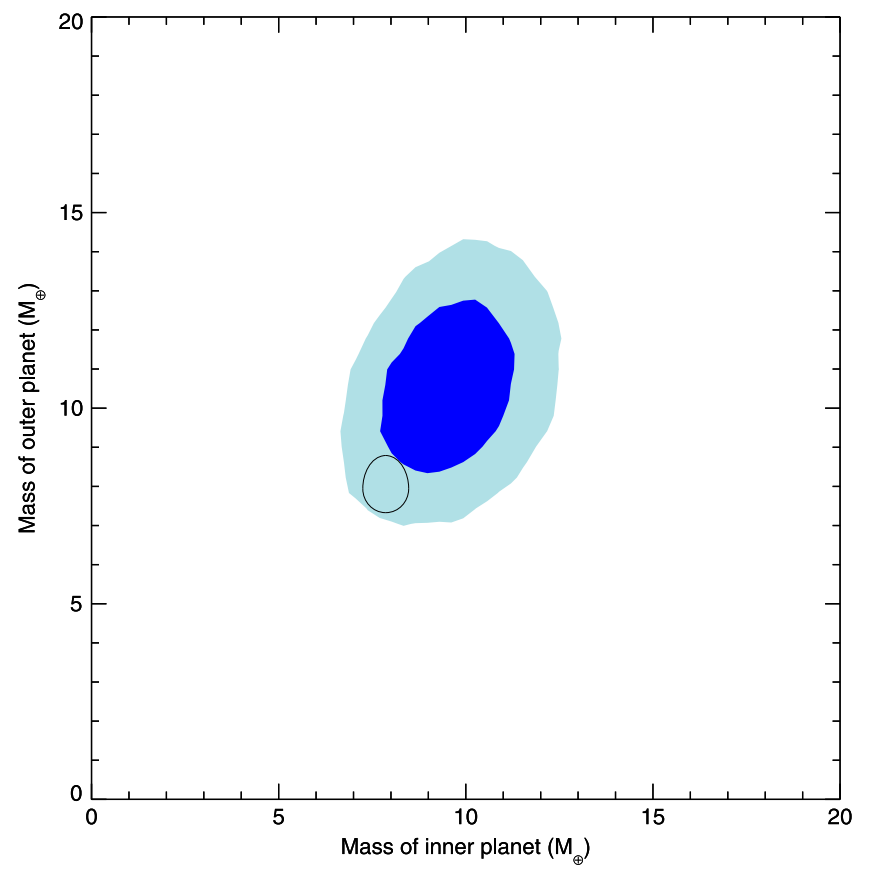

Figure 9. Comparison of Kepler-11 d/e resonant + chopping analysis. Black curve: $1 \sigma$ constraint from Lissauer et al. (2013). Dark (light) blue: 1(2) $\sigma$ constraint from 3:2 resonant term and chopping terms for both planets.

$Z_{\text {free }}=(0.054 \pm 0.017)+i(0.053 \pm 0.014)$. Since dissipation of eccentricities first damps the free eccentricities, it is interesting that the $Z_{\text {free }}$ here, though modest, is distinctly nonzero.

In general, because the chopping amplitude function $f_{i}^{j}(\alpha)$ is smaller in magnitude for larger values of $j$, the components with larger $j$ may not be measurable. However, if they are, they can be used to provide additional constraints on the masses as consistency checks. For example, in the case of Kepler $11 \mathrm{~d}$, the chopping signals with $j=1,2$ all independently constrain the mass of planet e, while the chopping signals of $j=1,3$ present in the TTVs of e all independently constrain the mass of planet d. For planet d, the inferred mass (assuming in this case a one solar mass star) is $10.4 \pm 1.8 M_{\oplus}(j=1)$ or $11.1 \pm 2.2 M_{\oplus}(j=3)$, compared to $7.86 \pm 0.61 M_{\oplus}$ in Lissauer et al. (2013). For planet e the inferred mass is $7.5 \pm 2.9 M_{\oplus}(j=1)$ or $10.5 \pm 2.3 M_{\oplus}(j=2)$, compared to $7.94 \pm 0.85 M_{\oplus}$ in Lissauer et al. (2013). Given that these estimates agree at the $(1-2) \sigma$ level, this indicates that the dynamical analysis yields masses that are consistent with the chopping amplitudes.

\subsection{Kepler 9}

In some cases, the Lithwick et al. (2012) formula alone can be used to determine the masses of the planets. In practice this requires that the amplitudes and phases of the TTVs must be measured with high accuracy.

The first system with detected TTVs is the Kepler-9 system (Holman et al. 2010), which consists of three planets, the outer two of which are close to $2: 1$ period commensurability with periods near 19 and 39 days, respectively. The outer pair is dynamically decoupled from the inner planet in that the inner planet does not measurably affect their TTVs. A recent dynamical analysis of Kepler-9 shows that the TTVs yield masses of the outer two planets of $m_{b}=45.1 \pm 1.5 M_{\oplus}$ and $m_{c}=31.0 \pm 1.0 M_{\oplus}$ (Dreizler \& Ofir 2014). We carried out a harmonic fit to the transit times for each planet, and find an excellent fit to planet Kepler-9c [d]'s transit times with four[six] harmonics of the period of Kepler-9d[c]. Figure 10 shows the TTVs for both planets along with the harmonic fit. The synodic chopping $(j=1)$ amplitude of the inner planet matches the phase and amplitude predicted based on the mass inferred by Dreizler \& Ofir (2014).

We carried out a Markov chain analysis on the set of transit times published by Dreizler \& Ofir (2014) for this system with the 2:1 resonant term and $j=1$ chopping, as well as additional harmonics with amplitudes that were not constrained by the physical parameters of the model: for the inner planet we added harmonics at $3 n_{2}, 4 n_{2}$, and $6 n_{2}$, while for the outer planet we added harmonics at $2 n_{1}, 3 n_{1}$, and $4 n_{1}$ to our model. We find masses of $m_{b}=49.9 \pm 2.6 M_{\oplus}$ and $m_{c}=35.6 \pm 1.8 M_{\oplus}$, similar to Dreizler \& Ofir (2014), albeit with larger uncertainties. When we remove the constraint on the amplitude of the chopping signal, we find comparable masses and uncertainties; we suspect that the reason for this is that in this case the amplitudes of the TTV are measured with sufficient precision that the $f$ and $g$ terms that occur in the first-order resonant TTV formula can be distinguished in amplitude. The imaginary component of the TTV is significant for both planets, so in this case one can break the degeneracy between the planet masses with just the resonant term. The amplitudes for the resonant term for both planets have four (well-measured) constraints, the real and imaginary amplitude for each planet, while there are four unknowns: the mass ratios for each planet, and the real and imaginary component of $Z_{\text {free }}$. This gives a unique solution, so the masses are well determined without the need for the chopping constraint.

For this system, then, the chopping signal is not required to determine the mass of the planets, but we can show that it is consistent with the masses inferred from the resonant terms alone. The measured mass of the outer planet predicts the amplitude of the $j=1$ synodic chopping signal of the inner planet, which only has $\sin \left(\lambda_{1}-\lambda_{2}\right)$ dependence. In Figure 11 we show that the measured sine amplitude of the inner chopping signal is consistent to $<0.2 \sigma$ with the predicted amplitude. The amplitude of the synodic chopping term gives a mass of the outer planet of $37.4 \pm 8.6 M_{\oplus}$, while the mass estimated from the resonant term is $35.6 \pm 1.8 M_{\oplus}$. In addition, the amplitude of the cosine term is consistent with zero at $<2 \sigma$, as it should be for the synodic chopping term.

When radial velocities are included in the analysis, a larger mass is derived for the planets, yielding about $55 M_{\oplus}$ for the outer planet (Dreizler \& Ofir 2014). This is inconsistent with the chopping signal, at about the $\approx 3 \sigma$ level (see green point in Figure 11) and inconsistent with the masses derived from resonant TTV alone. This discrepancy indicates that there is still some tension between the TTV data and the RV data, possibly due to RV jitter, additional planets (causing perturbations of the RV velocities), systematic errors in the transit times (perhaps due to star spot crossings), or, perhaps, simply statistical fluctuations. The fact that the resonant and chopping terms give similar estimates of the outer planet mass increases our confidence 


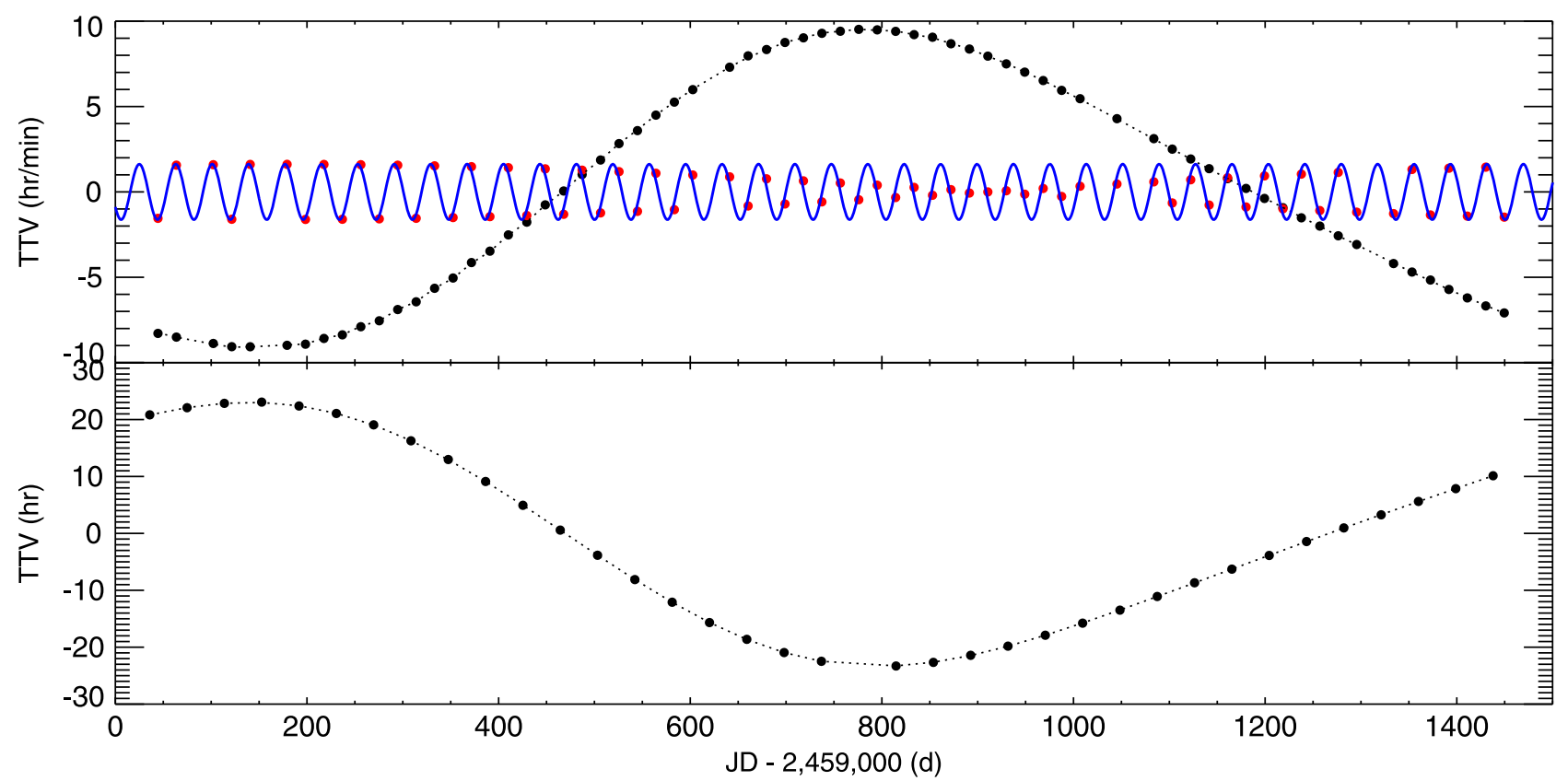

Figure 10. TTVs observed with Kepler for Kepler -9 (black filled circles), in units of hours. We have fit six (four) harmonics to the transit times of Kepler -9c(d), obtaining an excellent fit (dotted line). The synodic chopping component of our fit to the inner planet is plotted with red dots (in minutes), while the predicted synodic chopping signal based on the published dynamical fit is shown in blue (also in minutes).

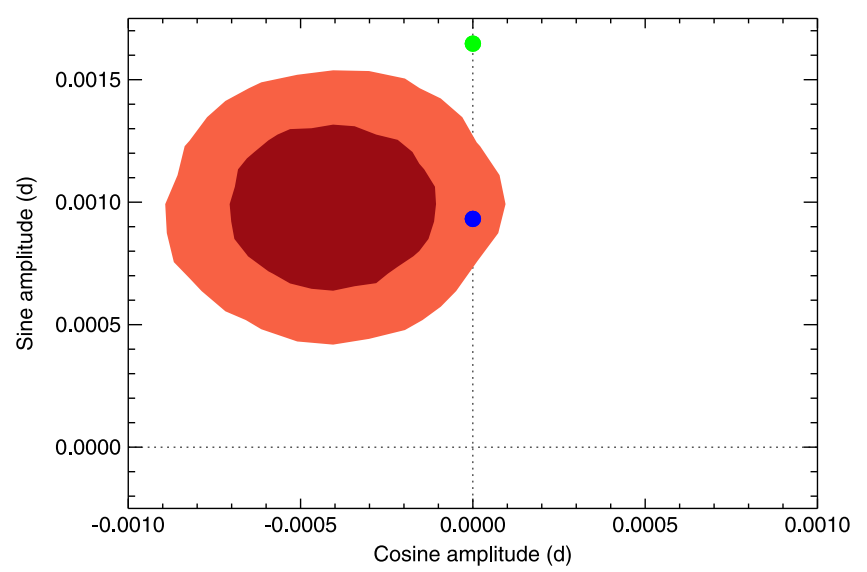

Figure 11. Measured amplitude of the synodic chopping $\sin \left(\lambda_{1}-\lambda_{2}\right)$ term in the fit to Kepler-9, as well as the $\cos \left(\lambda_{1}-\lambda_{2}\right)$ term, which is predicted to be zero; dark (light) red is $1(2) \sigma$ confidence region. These are compared to the $2: 1$ resonant mass for $m_{2}=35.6 M_{\oplus}$ (blue; from TTVs) and $m_{2}=55 M_{\oplus}$ (green; from RV).

that the transit timing analysis is not strongly affected by additional planets in this system.

For this system we also tried to use the amplitude of the $j=2$ chopping signal for the outer planet to constrain the mass of the inner planet. However, the amplitude is much too large to be due to chopping, and instead we believe is due to the 2:4 resonant term (which is of order $e^{2}$, but these planets are so close to $1: 2$, that the $1 / \Delta$ term compensates for this).

\subsection{Mass Precision of KOI-872c}

For planets that are not near a first-order mean-motion resonance $\left(P_{1} / P_{2} \approx\left(j_{R}-1\right) / j_{R}\right)$, the synodic chopping amplitude can provide the strongest constraint upon the planet masses. If there are a large number of transits, then the signal-

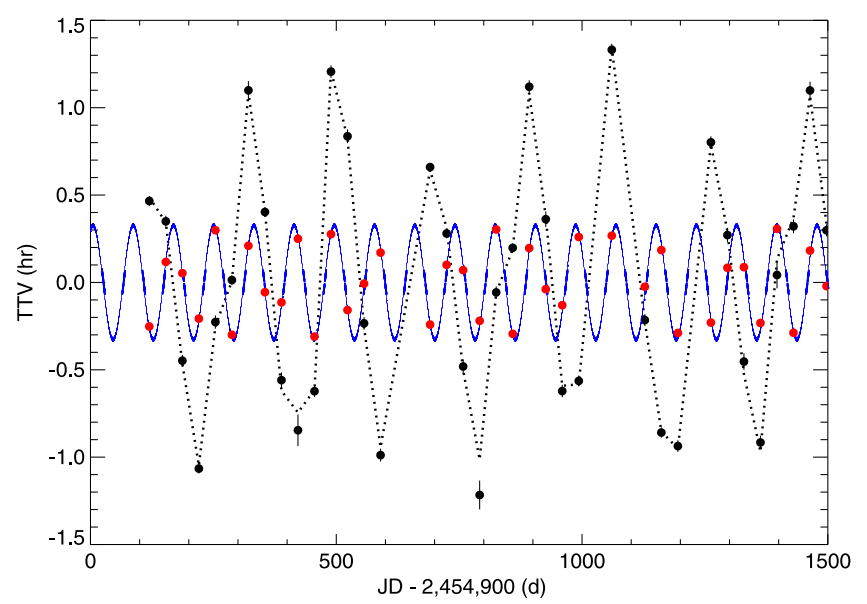

Figure 12. TTVs observed with Kepler for KOI-872 (black filled circles, with uncertainties). We have fit five harmonics to the transit times, obtaining an excellent fit (dotted line). The synodic chopping component of our fit is plotted with red dots, while the predicted synodic chopping signal based on the published dynamical fit is shown in blue.

to-noise of the planet mass ratio can be estimated with Equation (16).

The first non-transiting planet found with transit timing variations (with a unique identification of the perturbing planet's period) occurred in the system KOI-872 (Nesvorný et al. 2012), in which the period ratio of the two planets is close to 5:3. We carried out a harmonic analysis of the transit times of the inner planet with harmonics up to $5 n_{2}$, starting with the period of the perturbing planet, KOI-872c, from the published dynamical analysis. The synodic chopping signal, the coefficient of the $n_{2}$ term, was measured to have an amplitude of $a_{1}=0.0129 \pm 0.0004$ days for the sinusoidal component (detected at $32 \sigma$ ), and $b_{1}=-0.00049 \pm 0.00034$ for the cosine component (consistent with zero at $\approx 1.5 \sigma$ ). 
Applying the synodic chopping amplitude formula (Equation (10), with $j=1$ ), we find a mass ratio of: $m_{2} / M_{\star}=(3.81 \pm 0.12) \times 10^{-4}$. This compares favorably with the mass ratio measured from the full dynamical analysis (Nesvorný et al. 2012) of: $m_{2} / M_{\star}=\left(3.97_{-0.11}^{+0.15}\right) \times 10^{-4}$, with a similar magnitude uncertainty. Figure 12 shows the harmonic fit to the TTVs of KOI-872, with the synodic chopping signal shown with red points. The predicted synodic chopping based upon the dynamical solution is shown in blue, demonstrating agreement with the derived signal (albeit discrepant by $\approx 1.4 \sigma$ ).

The expected precision in the planet mass is given by Equation (16); for KOI-872 there are 37 transit times with a typical precision of 0.0015 days, 13 model parameters, giving an expected mass precision of $\sigma_{M_{2}}=1.25 \times 10^{-5}$, which matches well that found with the harmonic fits, and is close to the uncertainty found with the full dynamical analysis. This indicates that the timing precision along with the total number of transits observed and the number of free parameters fit can be used to forecast the mass measurement precision in the non-resonant case. A caveat is that this formula applies when chopping dominates the mass uncertainty, which may not be true for large eccentricities of the planets, which can cause higher order resonant terms to play a more important role.

\subsection{Predicting Planetary Mass Precision Inferred from the Synodic TTV}

The chopping effect potentially allows for mass measurements of planets with TTVs observed by a TESS-like mission because the synodic TTV, when unaliased with a resonant frequency, is a short-period effect. However, the amplitude of the effect is also considerably smaller than that due to the longtimescale resonant variations, and so more transits are needed to build up signal to noise. Here we consider a system with two planets with a period ratio of 1.5 , with an inner orbital period of 20 days. We assume that the system has been observed for one year (the baseline TESS will have for stars near the celestial poles), and assume that the timing uncertainty on the transit times of the inner planet are one minute.

In this case, $N_{\text {trans }} \approx 365.0(1 / 20.0+1 / 30.0) \approx 30$ and the formula in Equation (16) yields, for 10 free parameters and assuming a solar mass star, a mass precision of the outer planet based on the $j=1$ chopping of the inner planet of $\sim 1.3 M_{\oplus}$. The mass precision on the inner planet due to the $j=1$ chopping in the outer planet is $\sim 1 M_{\oplus}$. Wider pairs will have larger mass uncertainty since the function $f_{i}^{j}(\alpha)$ is smaller. For example, this same pair moved to the $2: 1$ resonance will allow an $\sim 6 M_{\oplus}$ mass uncertainty for the outer planet.

Note that the mass uncertainty scales like the inverse of the square of the orbital period, and, given an orbital period, like the inverse of the square of the observation time. Therefore, for a longer mission like PLATO, with an observational baseline of two or three years, the uncertainty on the mass of the perturbing planet will be smaller by a factor of $1 / \sqrt{2}$ or $1 / \sqrt{3}$, respectively, compared with that estimated for one year of data above.

\section{CONCLUSIONS}

In this paper we have written down expressions for transittiming variations in the plane-parallel limit, in the limit of zero free eccentricity of both planets, outside of resonance, and for timescales shorter than the secular timescale. Despite these assumptions, these terms have important consequences for analysis of transit-timing variations of multi-planet systems: 1) the TTVs have a dependence on $\sin \left[j\left(\lambda_{1}-\lambda_{2}\right)\right]$, for $j=1$ to $\infty ; 2)$ the amplitude of these terms only depends on the massratio of the perturbing planet to the star, and the semi-major axis ratio of these planets. Although other papers have presented formulae in the limit of zero-eccentricity (Agol et al. 2005; Nesvorný \& Vokrouhlický 2014b), this is the first time that the coefficients for each $j$ have been written down explicitly. This allows for harmonic analysis of transit times in terms of the period and phase of the perturbing planet; the coefficients of each harmonic can then be related to the planet mass ratios via these formulae (except for the harmonics affected by resonant terms). When the period and phase of the perturbing planet are known, then fitting for the harmonic coefficients (and the mean ephemeris) is a linear regression problem; thus a global solution can be found by simple matrix inversion, yielding a unique solution for the coefficients. This means that there cannot be multi-modal degeneracies in the derived masses of the planets. The amplitudes and uncertainties of these coefficients can then be translated into planet masses using the formulae given above. Alternatively the coefficients can computed from the physical properties of the planets (masses, eccentricity vector, and ephemerides), and then the model can be fit to the data with a nonlinear optimization or Markov chain, as we have carried out for several of the examples presented above.

In particular, we have highlighted the importance of the $j=1$ "synodic chopping" signal of the inner planet, which has a weak dependence on the eccentricities of the planets, and is not aliased with any first-order resonant terms. The formula for this term in particular has very little error even for very compact orbits. For the outer planet, the $j=1$ chopping signal can be used if the period ratios are distant from the 2:1 resonance, though if the transit times are precisely measured the synodic TTV resulting from values of $j \neq 1$ may be used to constrain the mass of the inner planet instead. In general, if the system is near or in a first-order resonance, with $P_{2} / P_{1} \approx\left(j_{R}-1\right) / j_{R}$, only the synodic TTV component with $j=j_{R}$ (inner planet) and $j=j_{R}-1$ (outer planet) will be altered; the formulae with $j$ not equal to these values will apply. Other limitations of this formula is that it cannot be directly used when more than one planet strongly perturbs the transiting planet, it breaks down for very massive planets $\left(m_{\text {planet }} \sim 10^{-3} M_{\star}\right)$ with period ratios less than 2 , and that as the period ratio approaches unity the error due to neglected eccentricity terms can become more important in the formula (though this does not apply to the $j=1$ term in the TTVs of the inner planet). Following Nesvorný \& Vokrouhlický (2014b), we conclude that in these regimes the chopping formula provides insight into mass measurements with TTVs even though the analytic formulae may be too approximate to be applied directly.

We have applied these formulae to existing transiting planet systems that have been analyzed in prior publications, recovering the mass measurements, but using harmonic fits and the analytic chopping/resonant expressions rather than full $\mathrm{N}$-body integrations. In some cases (KOI-872) this shows that the primary constraint on the mass of the planets comes from the synodic chopping signal. In other cases (KOI-1353c/d, 
Kepler 11d,e) the primary mass constraint comes from the combination of the first-order resonant signal and synodic chopping of the inner planet. In the case of Kepler-9, the primary constraint comes from the resonant signal, although the chopping component gives a consistent constraint on the mass of the outer planet.

In future applications, we expect that these formulae can be used for rapid fitting and estimation of TTVs, for rapid estimation of planet masses, for initialization of $\mathrm{N}$-body integration, for determining the requisite timing precision to measure planet masses with future follow-up observations of multi-planet transiting systems, and for forecast of TTVs. It should be possible to apply these formulae in systems of more than two planets using linear-combinations of the TTVs induced by more than one perturbing planet.

We would like to thank the referee, David Nesvorný, for helping us to clarify the text, and we would also like to thank Dan Fabrycky, Eric Ford, Matt Holman, Daniel Jontof-Hutter, Jack Lissauer, Jason Steffen, and the Kepler TTV group for helpful conversations. E.A. acknowledges funding by NSF Career Grant AST 0645416, NASA Astrobiology Institute's Virtual Planetary Laboratory, supported by NASA under cooperative agreement NNH05ZDA001C, and NASA through grants NNX13AF20G, NNX13AF62G, and an award issued by JPL/Caltech. K.M.D. acknowledges support from JCPA fellowship at Caltech. Work by K.M.D. during the summer of 2014 was supported by NASA under grant NNX09AB28G from the Kepler Participating Scientist Program and grants NNX09AB33G and NNX13A124G under the Origins program.

\section{APPENDIX A DERIVATION OF SYNODIC TTV}

Here we provide an alternate derivation of the synodic TTV to zeroth order in planetary eccentricities. We follow the method first described in Nesvorný \& Morbidelli (2008), though we derive the result using a Von Zeipel transformation instead of a Lie series approach. We seek a formula at zeroth order in eccentricities and inclinations and first-order in the parameter $m_{\text {planet }} / M_{\star}$.

As mentioned in the main text, the synodic TTV has been derived before, by Agol et al. (2005) and Nesvorný \& Vokrouhlický (2014b) (see also Nesvorný \& Vokrouhlický 2014a). The expressions differ slightly, in that those of Agol et al. (2005) and Nesvorný \& Vokrouhlický (2014b) contain extra terms in the form of constants, linear function of time, and periodic dependencies on $\lambda_{1}$ (inner planet) and $\lambda_{2}$ (outer planet). These terms do not contribute to the TTVs, and so the expressions agree in that they predict the same TTVs. We believe that the extra terms result because the derivations lead to different physical quantities. Our expression below applies to the timing variations (and orbital perturbations) compared to the average orbits. Since TTVs are calculated with reference to an average orbit, this makes intuitive sense. The expressions of Agol et al. (2005) and Nesvorný \& Vokrouhlický (2014b) instead calculate the deviations in the orbital elements at a given time with respect to those orbital elements at a reference time (not with respect to the average orbit).

For the outer planet, the reflex motion of the star dominates at large period ratios. This effect is accounted for in the equations in the appendix of Agol et al. (2005) and in the alternate derivation in this appendix, but is missing from the terms in Nesvorný \& Vokrouhlický (2014b). The Agol et al. 2005 calculation utilized heliocentric coordinates, while that of Nesvorný \& Vokrouhlický (2014b) used Jacobi coordinates and thus computed the TTVs of the outer planet relative to the center of mass, not relative to the star. Here we also employ Jacobi coordinates but correct for the reflex contribution afterwards.

TTVs are determined by taking an observed set of transit times and performing a linear fit to them, such that the TTV $\delta t_{n}$ can be written as

$$
\delta t_{n}=t_{n}-\left(t_{0}+n \bar{P}\right) .
$$

The slope $\bar{P}$ is the average time between successive transits during the observational baseline, or the average period over this timespan. In this sense, TTVs are the deviations from the transit times predicted by the average of the true (nonKeplerian) orbit.

Transits occur when the true longitude $\theta$ of the transiting planet is equal to a particular value. The expression for $\theta$ is, to first-order in eccentricity,

$$
\begin{aligned}
\theta & =f+\varpi \\
& =M+2 e \sin M+\varpi+O\left(e^{2}\right) \\
& =\lambda+2 e \sin (\lambda-\varpi)+O\left(e^{2}\right)
\end{aligned}
$$

where $e$ is the eccentricity, $f$ the true anomaly, $\varpi$ the longitude of periastron, $M$ the mean anomaly, and $\lambda=M+\varpi$ the mean longitude of the planet. Because we will perform the calculation using a Hamiltonian formalism, we now switch to canonical coordinates. Our variables will be

$$
\begin{aligned}
\Lambda_{i} & =m_{i} \sqrt{G M_{\star} a_{i}}, \quad \lambda_{i} \\
x_{i} & =\sqrt{2 P_{i}} \cos p_{i},
\end{aligned}
$$

where

$$
P_{i}=\Lambda_{i} \frac{e_{i}^{2}}{2}+O\left(e_{i}^{4}\right) \quad p_{i}=-\varpi_{i}
$$

where $i=1,2$ for the two planets, $m_{i}$ is the mass of the planet, $M_{\star}$ the mass of the star, and all orbital elements are Jacobi elements. Variables in the left columns are the canonical momenta, while those in the right columns are the conjugate coordinate. In terms of this canonical set, Equation (A.2) for $\theta$ becomes

$$
\theta=\lambda+\frac{2}{\sqrt{\Lambda}}(x \sin \lambda+y \cos \lambda) .
$$

We will perturb Equation (A.3) about the averaged orbit, and keep terms only at zeroth order in $x$ and $y$ :

$$
\delta \theta=\delta \lambda+\frac{2}{\sqrt{\Lambda}}(\delta x \sin \lambda+\delta y \cos \lambda)
$$

where as we will see the parameters $\delta$ are going to be of order $\epsilon=m_{\text {planet }} / M_{\star}$ and independent of eccentricities to lowest order. It is important to note that we define $\delta f \equiv f-\bar{f}$ for any arbitrary function $f$, where over-bar denotes a time-average. In that case,

$$
\delta \theta=-n \delta t
$$

where $\bar{n}$ is the average mean motion and $\delta t$ is the timing variation. Note that (1) we are consistent with the style that a 
negative timing variation corresponds to an "early" transit with $\delta \theta>0$ and (2) we can use the average mean motion because Equation (A.4) holds only at first-order in the perturbations; in the same sense, we can treat quantities without a $\delta$ on the righthand side as averaged variables as well.

The question now arises-How do we determine the perturbations to the average orbit $\delta \lambda, \delta x$ and $\delta y$ ?

The Hamiltonian for a system of two planets of mass $m_{i}$ orbiting a much more massive star of mass $M_{\star}$, written in Jacobi coordinates $\boldsymbol{r}_{i}$ and momenta $\boldsymbol{p}_{i}$, takes the form, to firstorder in combinations of $\epsilon=m_{\text {planet }} / M_{\star}$, of

$$
\begin{aligned}
H & =H_{0}+H_{1} \\
H_{0} & =H_{\text {Kepler }, 1}+H_{\text {Kepler }, 2} \\
H_{\text {Kepler }, i}= & \frac{p_{i}^{2}}{2 \tilde{m}_{i}}-\frac{G \tilde{M}_{i, \star} \tilde{m}_{i}}{\left|\boldsymbol{r}_{i}\right|} \\
H_{1}= & -G m_{1} m_{2}\left(\frac{1}{\left|\boldsymbol{r}_{1}-\boldsymbol{r}_{2}\right|}-\frac{\boldsymbol{r}_{2} \cdot \boldsymbol{r}_{1}}{\left|r_{2}\right|^{3}}\right) \\
& +O\left(\epsilon^{2}\right),
\end{aligned}
$$

where $\quad \tilde{M}_{2, \star}=M_{\star}\left(M_{\star}+m_{1}+m_{2}\right) /\left(M_{\star}+m_{1}\right), \quad \tilde{M}_{1, \star}=$ $\left(M_{\star}+m_{1}\right)$ and $\tilde{m}_{i}=m_{i}+O(\epsilon)$ denote Jacobi masses. Note that the perturbation $H_{1}$ takes the functional form of the disturbing function with an exterior perturber (Murray \& Dermott 1999). We set $\tilde{M}_{i, \star}=M_{\star}$ and also ignore the difference between Jacobi and physical masses. In the Keplerian piece $H_{0}$, this approximation corresponds to a (constant) change in the mean motions of the planets by order $\epsilon$, but since we are interested in TTVs this constant change does not matter. The correction between Jacobi and physical masses in the perturbation $H_{1}$ generates only $O\left(\epsilon^{2}\right)$ terms, and we ignore these.

When expressed in terms of the canonical set given in Equation (A) and Equation (A), the Hamiltonian takes the form

$$
H=H_{0}\left(\Lambda_{i}\right)+\epsilon_{1} H_{1}\left(\Lambda_{i}, P_{i}, \lambda_{i}, p_{i}\right)+O\left(\epsilon^{2}\right)
$$

where e.g., $\Lambda_{i}=\left(\Lambda_{1}, \Lambda_{2}\right), \epsilon_{1}=\frac{m_{1}}{M_{\star}}$, and

$$
\begin{aligned}
H_{0}= & -\frac{\mu_{1}}{2 \Lambda_{1}^{2}}-\frac{\mu_{2}}{2 \Lambda_{2}^{2}} \\
H_{1}= & -\frac{\mu_{2}}{\Lambda_{2}^{2}}\left[\sum_{j=-\infty}^{j=\infty} g_{j, 0}(\alpha) \cos j\left(\lambda_{1}-\lambda_{2}\right)\right. \\
& +\sum_{j=-\infty}^{j=\infty} g_{j, 27}(\alpha) \sqrt{\frac{2 P_{1}}{\Lambda_{1}}} \cos \left(j \lambda_{2}-(j-1) \lambda_{1}+p_{1}\right) \\
& +\sum_{j=-\infty}^{j=\infty} g_{j, 31}(\alpha) \sqrt{\frac{2 P_{2}}{\Lambda_{2}}} \\
& \left.\times \cos \left(j \lambda_{2}-(j-1) \lambda_{1}+p_{2}\right)\right]
\end{aligned}
$$

and $\mu_{i}=G^{2} M_{\star}^{2} m_{i}^{3}, \alpha=a_{1} / a_{2}, \sqrt{2 P / \Lambda}=e\left(1+O\left(e^{2}\right)\right)$, and $g_{j, 27}, g_{j, 31}$, and $g_{j, 0}$ are functions of Laplace coefficients with the indirect terms included (Murray \& Dermott 1999). ${ }^{7}$ The relevant

\footnotetext{
7 We could equivalently use $\epsilon_{2}$ instead of $\epsilon_{1}$ as our small parameter, the overall coefficient of $H_{1}$ would just change to $-\left(m_{2} / m_{1}\right)^{2} \mu_{1} / \Lambda_{2}^{2}$.
}

functions of Laplace coefficients can be written as

$$
\begin{aligned}
g_{j, 0}(\alpha) & =\frac{1}{2} b_{1 / 2}^{(j)}(\alpha)-\delta_{j, 1} \alpha \\
g_{j, 27}(\alpha) & =\frac{1}{2}\left(-2 j-\alpha \frac{d}{d \alpha}\right) b_{1 / 2}^{(j)}(\alpha)+\delta_{j, 1} \frac{3}{2} \alpha-\delta_{j,-1} \frac{1}{2} \alpha \\
g_{j, 31}(\alpha) & =\frac{1}{2}\left(-1+2 j+\alpha \frac{d}{d \alpha}\right) b_{1 / 2}^{(j-1)}(\alpha)-\delta_{j, 2} 2 \alpha \\
b_{1 / 2}^{(j)}(\alpha) & =\frac{1}{\pi} \int_{0}^{2 \pi} \frac{\cos (j \theta)}{\sqrt{1-2 \alpha \cos \theta+\alpha^{2}}} d \theta .
\end{aligned}
$$

In writing the gravitational potential in this form, we have assumed that $\alpha$ is not too close to unity, in which case Laplace coefficients converge slowly. For $\alpha \rightarrow 1$, the analogs of $g_{j, 0}, g_{j, 27}$, and $g_{j, 31}$ appearing with higher powers of eccentricity are larger and so the neglected terms in Equation (A.8) become more important.

We now convert the $\left(P_{i}, p_{i}\right)$ variables (Equation $\left.(\mathrm{A})\right)$ to the $\left(x_{i}, y_{i}\right)$ set (Equation $\left.(\mathrm{A})\right)$, and also set $j\left(\lambda_{1}-\lambda_{2}\right)=\psi_{j}$ and $j \lambda_{2}-(j-1) \lambda_{1}=\phi_{j}$. Then the perturbation Hamiltonian $H_{1}$ takes the form

$$
\begin{aligned}
H_{1}= & -\frac{\mu_{2}}{\Lambda_{2}^{2}}\left[\sum_{j=-\infty}^{j=\infty} g_{j, 0}(\alpha) \cos \psi_{j}\right. \\
& +\sum_{j=-\infty}^{j=\infty} g_{j, 27}(\alpha) \frac{1}{\sqrt{\Lambda_{1}}}\left(x_{1} \cos \phi_{j}-y_{1} \sin \phi_{j}\right) \\
& +\sum_{j=-\infty}^{j=\infty} g_{j, 31}(\alpha) \frac{1}{\sqrt{\Lambda_{2}}}\left(x_{2} \cos \phi_{j}\right. \\
& \left.\left.-y_{2} \sin \phi_{j}\right)\right] .
\end{aligned}
$$

If the system is not too close to any resonance, all periodic terms in the perturbation $H_{1}$ are short-period, and an average over the (fast) angles $\lambda_{1}-\lambda_{2}$ and e.g., $\lambda_{2}$ leads to the averaged Hamiltonian $\bar{H}$, which is only a function of $\left(\bar{\Lambda}_{i}\right)$ (the assumption that all terms are short-period will be discussed at the end of this section). This averaged Hamiltonian takes the same function form of $H_{0}\left(\bar{\Lambda}_{i}\right)$, and so the evolution of the averaged orbits is "Keplerian": only the $\bar{\lambda}_{i}$ vary, and they do so at a constant rate given by $\bar{n}_{i}=\sqrt{G M_{\star} / \bar{a}_{i}^{3}}$. These are the averaged orbits we are interested in for calculating TTVs, and hence we need to determine a canonical transformation which turns $H$ into $\bar{H}$ and hence also changes exact variables into averaged ones.

This canonical transformation requires removing the shortperiod terms via a generating function so that they do not appear in the averaged Hamiltonian. The deviation between the averaged and full trajectories is of order $\epsilon$, and hence the canonical transformation between the two sets of variables should be very near the identity transformation. Therefore, we seek a generating function of Type 2 , which determines a nearidentity transformation that removes the short-period terms. We will write this generating function as

$$
F_{2}\left(\lambda_{i}, y_{i}, \bar{\Lambda}_{i}, \bar{x}_{i}\right)=\lambda_{i} \bar{\Lambda}_{i}+y_{i} \bar{x}_{i}+\epsilon_{1} f\left(\lambda_{i}, y_{i}, \bar{\Lambda}_{i}, \bar{x}_{i}\right)
$$

where $f$ is yet to be determined.

Again the variables with over-bars are the averaged canonical coordinates and momenta, while those without 
correspond to the unaveraged variables. The old and new variables are related as

$$
\begin{gathered}
\Lambda_{i}=\frac{\partial F_{2}}{\partial \lambda_{i}}=\bar{\Lambda}_{i}+\epsilon_{1} \frac{\partial f}{\partial \lambda_{i}} \\
x_{i}=\frac{\partial F_{2}}{\partial y_{i}}=\bar{x}_{i}+\epsilon_{1} \frac{\partial f}{\partial y_{i}} \\
\bar{\lambda}_{i}=\frac{\partial F_{2}}{\partial \bar{\Lambda}_{i}}=\lambda_{i}+\epsilon_{1} \frac{\partial f}{\partial \bar{\Lambda}_{i}} \\
\bar{y}_{i}=\frac{\partial F_{2}}{\partial \bar{x}_{i}}=y_{i}+\epsilon_{1} \frac{\partial f}{\partial \bar{x}_{i}}
\end{gathered}
$$

so that the differences we are seeking for Equation (A.4) are

$$
\begin{gathered}
\delta \lambda_{i}=\lambda_{i}-\bar{\lambda}_{i}=-\epsilon_{1} \frac{\partial f}{\partial \bar{\Lambda}_{i}} \\
\delta x_{i}=x_{i}-\bar{x}_{i}=\epsilon_{1} \frac{\partial f}{\partial y_{i}} \\
\delta y_{i}=y_{i}-\bar{y}_{i}=-\epsilon_{1} \frac{\partial f}{\partial \bar{x}_{i}} .
\end{gathered}
$$

Then, the expression for $\delta \theta_{i}$ is simply

$$
\delta \theta_{i}=\epsilon_{1}\left[-\frac{\partial f}{\partial \bar{\Lambda}_{i}}+\frac{2}{\sqrt{\Lambda_{i}}}\left(\frac{\partial f}{\partial y_{i}} \sin \lambda_{i}-\frac{\partial f}{\partial \bar{x}_{i}} \cos \lambda_{i}\right)\right]
$$

where we can evaluate the function $f\left(\lambda_{i}, y_{i}, \bar{\Lambda}_{i}, \bar{x}_{i}\right)$ as $f\left(\bar{\lambda}_{i}, \bar{y}_{i}, \bar{\Lambda}_{i}, \bar{x}_{i}\right)$ because the difference between the averaged and unaveraged variables is already of order $\epsilon$.

The problem now reduces to finding the function $f$. The new (averaged) Hamiltonian is $\bar{H}\left(\bar{\Lambda}_{i}, \bar{x}_{i}, \bar{\lambda}_{i}, \bar{y}_{i}\right)=$ $H\left[\bar{\Lambda}_{i}\left(\Lambda_{i}, x_{i}, \lambda_{i}, y_{i}\right), \bar{x}_{i}\left(\Lambda_{i}, x_{i}, \lambda_{i}, y_{i}\right), \bar{\lambda}_{i}\left(\Lambda_{i}, x_{i}, \lambda_{i}, y_{i}\right), \bar{y}_{i}\right.$ $\left.\left.\left(\Lambda_{i}, x_{i}, \lambda_{i}, y_{i}\right)\right)\right]$ since the generating function is time independent. To first-order in $\epsilon_{1}$,

$$
\begin{aligned}
\bar{H}\left(\bar{\Lambda}_{i}, \bar{x}_{i}, \bar{\lambda}_{i}, \bar{y}_{i}\right)= & H_{0}\left(\bar{\Lambda}_{1}+\epsilon_{1} \frac{\partial f}{\partial \lambda_{1}}, \bar{\Lambda}_{2}+\epsilon_{1} \frac{\partial f}{\partial \lambda_{2}}\right) \\
& +\epsilon_{1} H_{1}\left(\bar{\Lambda}_{i}, \bar{x}_{i}, \bar{\lambda}_{i}, \bar{y}_{i}\right) \\
& =H_{0}\left(\bar{\Lambda}_{i}\right)+\epsilon_{1}\left(\frac{\partial H_{0}}{\partial \Lambda_{1}} \frac{\partial f}{\partial \lambda_{1}}\right. \\
& \left.+\frac{\partial H_{0}}{\partial \Lambda_{2}} \frac{\partial f}{\partial \lambda_{2}}\right)\left.\right|_{\left(\Lambda_{i}, x_{i}, \lambda_{i}, y_{i}\right)=\left(\bar{\Lambda}_{i}, \bar{x}_{i}, \bar{\lambda}_{i}, \bar{y}_{i}\right)} \\
& +\epsilon_{1} H_{1}\left(\bar{\Lambda}_{i}, \bar{x}_{i}, \bar{\lambda}_{i}, \bar{y}_{i}\right) \\
& =H_{0}\left(\bar{\Lambda}_{i}\right)+\left.\epsilon_{1}\left(\bar{n}_{1} \frac{\partial f}{\partial \lambda_{1}}+\bar{n}_{2} \frac{\partial f}{\partial \lambda_{2}}\right)\right|_{\left(\Lambda_{i}, x_{i}, \lambda_{i}, y_{i}\right)=\left(\bar{\Lambda}_{i}, \bar{x}_{i}, \bar{\lambda}_{i}, \bar{y}_{i}\right)} \\
& +\epsilon_{1} H_{1}\left(\bar{\Lambda}_{i}, \bar{x}_{i}, \bar{\lambda}_{i}, \bar{y}_{i}\right)
\end{aligned}
$$

where $\bar{n}_{i}=\mu_{i}^{2} / \bar{\Lambda}_{i}^{3}$ is the average mean motion of planet $i$.

Now, we would like this averaged Hamiltonian to be equal to

$$
\bar{H}=H_{0}\left(\bar{\Lambda}_{i}\right),
$$

i.e., with all short period terms removed. We must choose the function $f$ to satisfy the relation

$$
\left(\bar{n}_{1} \frac{\partial f}{\partial \lambda_{1}}+\bar{n}_{2} \frac{\partial f}{\partial \lambda_{2}}\right)+H_{1}=0 .
$$

Given an $H_{1}$ that takes the functional form $H_{1}=A \cos \theta+B \sin \theta$, where $A$ and $B$ are coefficients independent of $\lambda_{i}$, and $\theta=p \lambda_{1}+q \lambda_{2}$, for any integers $p$ and $q$, the solution $f$ to Equation (A.17) is

$$
f=\frac{1}{\dot{\theta}}[-A \sin \theta+B \cos \theta]
$$

where $\dot{\theta}=p \bar{n}_{1}+q \bar{n}_{2}$. Therefore, the solution to Equation (A.19) for the full $H_{1}$ given in Equation (A.10) is

$$
\begin{aligned}
f= & \frac{\mu_{2}}{\bar{\Lambda}_{2}^{2}} \sum_{j}\left(\frac{g_{j, 0}(\bar{\alpha}) \sin \psi_{j}}{j\left(\bar{n}_{1}-\bar{n}_{2}\right)}\right. \\
& +\frac{g_{j, 27}(\bar{\alpha})}{\sqrt{\bar{\Lambda}_{1}}\left(j \bar{n}_{2}-(j-1) \bar{n}_{1}\right)}\left(\bar{x}_{1} \sin \phi_{j}+y_{1} \cos \phi_{j}\right) \\
& +\frac{g_{j, 31}(\bar{\alpha})}{\sqrt{\bar{\Lambda}_{2}}\left(j \bar{n}_{2}-(j-1) \bar{n}_{1}\right)} \\
& \left.\times\left(\bar{x}_{2} \sin \phi_{j}+y_{2} \cos \phi_{j}\right)\right) .
\end{aligned}
$$

Here, $\sum_{j}$ denotes a sum over all possible negative and positive integers. Note, however, that the first term in A.19 dependent on $g_{j 0}$ diverges when $j=0$. This value of $j$ should not be included in the sum, for this term only. It corresponds to a constant in the interaction Hamiltonian, independent of the orbital angles, and so its derivative, which would appear in the generating function, is zero. We will ignore this term, since it only leads to a constant change in the mean motion of the planets. At this point, we will also stop denoting averaged variables with over-bars, since at the order we are working in $\epsilon$ these two are equivalent. Then,

$$
\begin{aligned}
\frac{\partial f}{\partial \bar{\Lambda}_{1}}= & \frac{\mu_{2}}{\Lambda_{2}^{2}} \sum_{j}\left(\frac{d g_{j, 0}(\alpha)}{d \alpha} \frac{\partial \alpha / \partial \Lambda_{1}}{j\left(n_{1}-n_{2}\right)}\right. \\
& \left.-g_{j, 0}(\alpha) \frac{\partial n_{1} / \partial \Lambda_{1}}{j\left(n_{1}-n_{2}\right)^{2}}\right) \sin \psi_{j}+O(e) \\
= & \frac{\mu_{2}}{\Lambda_{2}^{2}} \sum_{j}\left(\frac{d g_{j, 0}(\alpha)}{d \alpha} \frac{2 \alpha / \Lambda_{1}}{j\left(n_{1}-n_{2}\right)}\right. \\
& \left.-g_{j, 0}(\alpha) \frac{-3 n_{1} / \Lambda_{1}}{j\left(n_{1}-n_{2}\right)^{2}}\right) \sin \psi_{j}+O(e) \\
= & \frac{n_{2}}{n_{1}} \frac{\Lambda_{2}}{\Lambda_{1}} \sum_{j}\left(\frac{d g_{j, 0}(\alpha)}{d \alpha} \frac{2 \alpha n_{1}}{j\left(n_{1}-n_{2}\right)}\right. \\
& \left.+g_{j, 0}(\alpha) \frac{3 n_{1}^{2}}{j\left(n_{1}-n_{2}\right)^{2}}\right) \sin \psi_{j}+O(e) \\
= & \frac{m_{2}}{m_{1}} \alpha \sum_{j}\left(\frac{d g_{j, 0}(\alpha)}{d \alpha} \frac{2 \alpha}{\beta}+g_{j, 0}(\alpha) \frac{3 j}{\beta^{2}}\right) \sin \psi_{j} \\
& +O(e)
\end{aligned}
$$


where $\beta=j\left(n_{1}-n_{2}\right) / n_{1}$, and

$$
\begin{aligned}
\frac{\partial f}{\partial \bar{\Lambda}_{2}}= & \frac{\mu_{2}}{\Lambda_{2}^{2}} \sum_{j}\left(\frac{d g_{j, 0}(\alpha)}{d \alpha} \frac{\partial \alpha / \partial \Lambda_{2}}{j\left(n_{1}-n_{2}\right)}+g_{j, 0}(\alpha) \frac{\partial n_{2} / \partial \Lambda_{2}}{j\left(n_{1}-n_{2}\right)^{2}}\right) \\
& \times \sin \psi_{j}-2 n_{2} \sum_{j} \frac{g_{j, 0}(\alpha)}{j\left(n_{1}-n_{2}\right)} \sin \psi_{j}+O(e) \\
= & n_{2} \sum_{j}\left(\frac{d g_{j, 0}(\alpha)}{d \alpha} \frac{-2 \alpha}{j\left(n_{1}-n_{2}\right)}\right. \\
& \left.-g_{j, 0}(\alpha) \frac{3 n_{2}}{j\left(n_{1}-n_{2}\right)^{2}}-2 \frac{g_{j, 0}(\alpha)}{j\left(n_{1}-n_{2}\right)}\right) \sin \psi_{j}+O(e) \\
= & -\sum_{j}\left(\frac{d g_{j, 0}(\alpha)}{d \alpha} \frac{2 \alpha}{\kappa}+g_{j, 0}(\alpha) \frac{3 j}{\kappa^{2}}\right. \\
& \left.+2 \frac{g_{j, 0}(\alpha)}{\kappa}\right) \sin \psi_{j}+O(e)
\end{aligned}
$$

where $\kappa=j\left(n_{1}-n_{2}\right) / n_{2}$.

We now calculate the changes in $x_{i}$ and $y_{i}$ (again ignoring the difference between averaged and unaveraged variables at this order in $\epsilon$ ):

$$
\begin{aligned}
\frac{\partial f}{\partial y_{1}} & =\frac{\mu_{2}}{\Lambda_{2}^{2}} \sum_{j} \frac{g_{j, 27}(\alpha)}{\sqrt{\Lambda_{1}}\left(j n_{2}-(j-1) n_{1}\right)} \cos \phi_{j} \\
\frac{\partial f}{\partial y_{2}} & =\frac{\mu_{2}}{\Lambda_{2}^{2}} \sum_{j} \frac{g_{j, 31}(\alpha)}{\sqrt{\Lambda_{2}}\left(j n_{2}-(j-1) n_{1}\right)} \cos \phi_{j} \\
\frac{\partial f}{\partial x_{1}} & =\frac{\mu_{2}}{\Lambda_{2}^{2}} \sum_{j} \frac{g_{j, 27}(\alpha)}{\sqrt{\Lambda_{1}}\left(j n_{2}-(j-1) n_{1}\right)} \sin \phi_{j} \\
\frac{\partial f}{\partial x_{2}} & =\frac{\mu_{2}}{\Lambda_{2}^{2}} \sum_{j} \frac{g_{j, 31}(\alpha)}{\sqrt{\Lambda_{2}}\left(j n_{2}-(j-1) n_{1}\right)} \sin \phi_{j} .
\end{aligned}
$$

Ultimately, we want the combinations

$$
\begin{aligned}
Z_{i} & =\frac{2}{\sqrt{\Lambda_{i}}}\left(\delta x_{i} \sin \lambda_{i}-\delta y_{i} \cos \lambda_{i}\right) \\
& =\frac{2 \epsilon_{1}}{\sqrt{\Lambda_{i}}}\left(\frac{\partial f}{\partial y_{i}} \sin \lambda_{i}-\frac{\partial f}{\partial x_{i}} \cos \lambda_{i}\right)
\end{aligned}
$$

For the inner planet, this is

$$
\begin{aligned}
Z_{1} & =\epsilon_{1} \frac{\mu_{2}}{\Lambda_{2}^{2}} \frac{2}{\Lambda_{1}} \sum_{j} \frac{g_{j, 27}(\alpha)}{j n_{2}-(j-1) n_{1}}\left(\cos \phi_{j} \sin \lambda_{1}-\sin \phi_{j} \cos \lambda_{1}\right) \\
& =2 \epsilon_{1} n_{2} \frac{\Lambda_{2}}{\Lambda_{1}} \sum_{j} \frac{g_{j, 27}(\alpha)}{j n_{2}-(j-1) n_{1}} \sin \left(\lambda_{1}-\theta_{j}\right) \\
& =2 \epsilon_{1} \frac{n_{2}}{n_{1}} \frac{m_{2}}{m_{1}} \alpha^{-1 / 2} \sum_{j} \frac{g_{j, 27}(\alpha) n_{1}}{j n_{2}-(j-1) n_{1}} \sin \psi_{j} \\
& =2 \frac{m_{2}}{m_{1}} \epsilon_{1} \alpha \sum_{j} \frac{g_{j, 27}(\alpha)}{1-\beta} \sin \psi_{j} .
\end{aligned}
$$

Note that only a synodic dependence of $\psi_{j}$ remains. At zeroth order in eccentricity, there are no first-order (and possibly resonant) angles $\phi_{j}$, although we needed these terms to compute the final result. The final expression for $\delta \theta_{1}$ is given by

$$
\begin{aligned}
& -n_{1} \delta t_{1}=\delta \theta_{1}=Z_{1}+\delta \lambda_{1} \\
& \delta \theta_{1}=\frac{m_{2}}{m_{1}} \alpha \epsilon_{1} \sum_{j}\left(2 \frac{g_{j, 27}(\alpha)}{1-\beta}\right. \\
& \left.-\frac{d g_{j, 0}(\alpha)}{d \alpha} \frac{2 \alpha}{\beta}-g_{j, 0}(\alpha) \frac{3 j}{\beta^{2}}\right) \sin \psi_{j} \\
& \delta t_{1}=\frac{P_{1}}{2 \pi} \epsilon_{2} \alpha \sum_{j=-\infty}^{j=\infty}\left(-2 \frac{g_{j, 27}(\alpha)}{1-\beta}\right. \\
& \left.+\frac{d g_{j, 0}(\alpha)}{d \alpha} \frac{2 \alpha}{\beta}+g_{j, 0}(\alpha) \frac{3 j}{\beta^{2}}\right) \sin \psi_{j} \\
& \delta t_{1}=\frac{P_{1}}{2 \pi} \epsilon_{2} \alpha \sum_{j=1}^{\infty}\left(\left[\frac{d g_{j, 0}(\alpha)}{d \alpha}+\frac{d g_{-j, 0}(\alpha)}{d \alpha}\right] \frac{2 \alpha}{\beta}\right. \\
& +\left[g_{j, 0}(\alpha)+g_{-j, 0}(\alpha)\right] \frac{3 j}{\beta^{2}} \\
& \left.+2\left[-\frac{g_{j, 27}(\alpha)}{1-\beta}+\frac{g_{-j, 27}(\alpha)}{1+\beta}\right]\right) \sin \psi_{j} \\
& \delta t_{1}=\frac{P_{1}}{2 \pi} \epsilon_{2} \alpha \sum_{j=1}^{\infty}\left(\frac{2}{\beta}\left[D_{\alpha} b_{1 / 2}^{(j)}(\alpha)-\alpha \delta_{j, 1}\right]\right. \\
& +\frac{3 j}{\beta^{2}}\left[b_{1 / 2}^{(j)}(\alpha)-\alpha \delta_{j, 1}\right] \\
& +\frac{2}{\left(1-\beta^{2}\right)}\left[\left(2 j b_{1 / 2}^{(j)}(\alpha)+\beta D_{\alpha} b_{1 / 2}^{(j)}(\alpha)\right.\right. \\
& \left.\left.-\alpha \delta_{j, 1}(2+\beta)\right]\right) \sin \psi_{j} \\
& \delta t_{1}=\frac{P_{1}}{2 \pi} \epsilon_{2} \alpha \\
& \times \frac{\left(\begin{array}{c}
2 \beta D_{\alpha} b_{1 / 2}^{(j)}(\alpha)+j\left(3+\beta^{2}\right) b_{1 / 2}^{(j)}(\alpha) \\
-\alpha \delta_{j, 1}\left(\beta^{2}+2 \beta+3\right)
\end{array}\right)}{\beta^{2}\left(1-\beta^{2}\right)} \\
& \times \sin \psi_{j}
\end{aligned}
$$

where we have neglected the constant $j=0$ contribution, defined $D_{\alpha}(\cdot)=\alpha \partial(\cdot) / \partial \alpha$, and taken advantage of the fact that as $j \rightarrow-j, \beta$ changes sign and the Laplace coefficients $b_{1 / 2}^{(j)}$ and their derivatives do not. Therefore,

$$
\begin{aligned}
\delta t_{1} & =\frac{P_{1}}{2 \pi} \epsilon_{2} \sum_{j=1}^{\infty} f_{1}^{(j)}(\alpha) \sin \psi_{j} \\
f_{1}^{(j)}(\alpha) & =\frac{\left(\begin{array}{c}
2 \beta D_{\alpha} b_{1 / 2}^{(j)}(\alpha)+j\left(3+\beta^{2}\right) b_{1 / 2}^{(j)}(\alpha) \\
-\alpha \delta_{j, 1}\left(\beta^{2}+2 \beta+3\right)
\end{array}\right)}{\beta^{2}\left(1-\beta^{2}\right)} .
\end{aligned}
$$


For the outer planet, the combination $Z_{2}$ is

$$
\begin{aligned}
Z_{2}= & \epsilon_{1} \frac{\mu_{2}}{\Lambda_{2}^{2}} \frac{2}{\Lambda_{2}} \sum_{j} \frac{g_{j, 31}(\alpha)}{j n_{2}-(j-1) n_{1}} \\
& \times\left(\cos \phi_{j} \sin \lambda_{2}-\sin \phi_{j} \cos \lambda_{2}\right) \\
= & 2 \epsilon_{1} n_{2} \sum_{j} \frac{g_{j, 31}(\alpha)}{j n_{2}-(j-1) n_{1}} \sin \left(\lambda_{2}-\phi_{j}\right) \\
= & 2 \epsilon_{1} n_{2} \sum_{j} \frac{g_{j, 31}(\alpha) n_{1}}{j n_{2}-(j-1) n_{1}} \sin \psi_{j-1} \\
= & 2 \epsilon_{1} n_{2} \sum_{k} \frac{g_{k+1,31}(\alpha)}{(1+k) n_{2}-k n_{1}} \sin \psi_{k} \\
= & 2 \epsilon_{1} n_{2} \sum_{j} \frac{g_{j+1,31}(\alpha)}{(1+j) n_{2}-j n_{1}} \sin \psi_{j} \\
= & 2 \epsilon_{1} \sum_{j} \frac{g_{j+1,31}(\alpha)}{1-\kappa} \sin \psi_{j} .
\end{aligned}
$$

Again, only the synodic angle $\psi_{j}$ remains. Then the expression for $\delta t_{2}$ is

$$
\begin{aligned}
\delta t_{2}= & -\frac{P_{2}}{2 \pi} \epsilon_{1} \sum_{j=-\infty}^{j=\infty}\left(\frac{d g_{j, 0}(\alpha)}{d \alpha} \frac{2 \alpha}{\kappa}+g_{j, 0}(\alpha) \frac{3 j}{\kappa^{2}}\right. \\
& \left.+2 \frac{g_{j, 0}(\alpha)}{\kappa}+2 \frac{g_{j+1,31}(\alpha)}{1-\kappa}\right) \sin \psi_{j} \\
= & -\frac{P_{2}}{2 \pi} \epsilon_{1} \sum_{j=1}^{j=\infty}\left(\left[\frac{d g_{j, 0}(\alpha)}{d \alpha}+\frac{d g_{-j, 0}(\alpha)}{d \alpha}\right] \frac{2 \alpha}{\kappa}\right. \\
& +\left[g_{j, 0}(\alpha)+g_{-j, 0}(\alpha)\right]\left[\frac{3 j}{\kappa^{2}}+\frac{2}{\kappa}\right) \\
& \left.+2\left[\frac{g_{j+1,31}(\alpha)}{1-\kappa}-\frac{g_{-j+1,31}(\alpha)}{1+\kappa}\right]\right) \sin \psi_{j} \\
= & -\frac{P_{2}}{2 \pi} \epsilon_{1} \sum_{j=1}^{j=\infty}\left(\left[D_{\alpha} b_{1 / 2}^{(j)}(\alpha)-\alpha \delta_{j, 1}\right] \frac{2}{\kappa}\right. \\
& +\left[b_{1 / 2}^{(j)}(\alpha)-\alpha \delta_{j, 1}\right]\left(\frac{3 j+2 \kappa}{\kappa^{2}}\right)+2 \\
& \left.\times \frac{(2 j+\kappa) b_{1 / 2}^{(j)}(\alpha)+\kappa D_{\alpha} b_{1 / 2}^{(j)}(\alpha)-2 \alpha \delta_{j+1,2}(1+\kappa)}{1-\kappa^{2}}\right)
\end{aligned}
$$$$
\times \sin \psi_{j}
$$$$
=\frac{P_{2}}{2 \pi} \epsilon_{1} \sum_{j=1}^{j=\infty} \sin \psi_{j} \frac{\left(\begin{array}{c}
j\left(\kappa^{2}+3\right) b_{1 / 2}^{(j)}(\alpha)+2 \kappa\left(b_{1 / 2}^{(j)}(\alpha)\right. \\
\left.+D_{\alpha} b_{1 / 2}^{(j)}(\alpha)\right)-\alpha \delta_{j, 1}\left(\kappa^{2}+4 \kappa+3\right)
\end{array}\right)}{\kappa^{2}\left(\kappa^{2}-1\right)} .
$$

However, this expression must be modified, since this corresponds to the orbit of the outer planet in Jacobi coordinates, about the center of mass of the inner planet and star subsystem. The correction to this is given in Agol et al. (2005) as

$$
\delta t_{2, \text { astrocentric }}=\delta t_{2, \text { jacobi }}-\frac{P_{2}}{2 \pi} \epsilon_{1} \alpha \sin \psi_{1}+O\left(e_{1}\right) .
$$

We add this to the indirect terms $I$ proportional to $\delta_{j, 1}$ and rearrange

$$
\begin{aligned}
I & =-\delta_{j, 1} \frac{\alpha\left(\kappa^{2}+4 \kappa+3\right)+\alpha\left(\kappa^{2}\left(\kappa^{2}-1\right)\right)}{\kappa^{2}\left(\kappa^{2}-1\right)} \\
& =-\delta_{j, 1} \frac{\alpha\left(\kappa^{4}+4 \kappa+3\right)}{\kappa^{2}\left(\kappa^{2}-1\right)} \\
& =-\delta_{j, 1} \alpha^{-2}\left(6+\alpha^{-3}-4 \alpha^{-3 / 2}\right) \kappa^{2}\left(\kappa^{2}-1\right) \\
& =-\delta_{j, 1} \alpha^{-2} \frac{\left(\kappa^{2}-2 \kappa+3\right)}{\kappa^{2}\left(\kappa^{2}-1\right)}
\end{aligned}
$$

so that we can write

$$
\begin{aligned}
\delta t_{2} & =\frac{P_{2}}{2 \pi} \epsilon_{1} \sum_{j=1}^{\infty} f_{2}^{(j)}(\alpha) \sin \psi_{j} \\
f_{2}^{(j)}(\alpha) & =\frac{\left(\begin{array}{c}
j\left(\kappa^{2}+3\right) b_{1 / 2}^{(j)}(\alpha)+2 \kappa\left(b_{1 / 2}^{(j)}(\alpha)\right. \\
\left.+D_{\alpha} b_{1 / 2}^{(j)}(\alpha)\right) \\
-\alpha^{-2} \delta_{j, 1}\left(\kappa^{2}-2 \kappa+3\right)
\end{array}\right)}{\kappa^{2}\left(\kappa^{2}-1\right)} .
\end{aligned}
$$

We return now to one of the principal assumptions made in deriving these formulae, namely that all periodic terms in the Hamiltonian are short-period. Near and in the $j_{R}: j_{R}-1$ resonance this assumption breaks down. Indeed, the generating function itself does not converge because certain terms possess small denominators (the resonant combination of orbital frequencies). However, if we exclude those terms in the generating function, and proceed as we did in the derivation, the resulting TTV expression will still encompass the shortperiod effect. Moreover, they should be identical to our expressions but without the synodic terms with $j=j_{R}$ for the inner planet and $j=j_{R}-1$ for the outer planet. In other words, the synodic TTV expressions we derived should apply equally well for resonant and non-resonant systems with the exception that for systems near and in resonance the formula for the synodic harmonic aliased to the resonant frequency will be incorrect.

\section{APPENDIX B CONVERGENCE OF SYNODIC TTV SUMS}

Here we address the question of the convergence of the sums that appear in the synodic TTV expressions. We assume that the system is not near a resonance so that there are no small denominators and consider the limit of large $j$. The Laplace coefficients can be written as (Murray \& Dermott 1999)

$$
\begin{aligned}
\frac{1}{2} b_{s}^{(j)}(\alpha)= & \frac{s(s+1) \ldots(s+j-1)}{j !} \alpha^{j}\left[1+\frac{s(s+j)}{1(j+1)} \alpha^{2}\right. \\
& +\frac{s(s+1)(s+j)(s+j+1)}{1 \cdot 2(j+1)(j+2)} \alpha^{4} \\
& \left.+O\left(\alpha^{6}\right)\right]
\end{aligned}
$$


In the limit of large $j$ and $\alpha<1$, this has a leading coefficient that scales as

$$
b_{s}^{(j)}(\alpha) \propto \alpha^{j}
$$

and therefore

$$
D_{\alpha} b_{s}^{(j)}(\alpha) \propto j \alpha^{j} .
$$

In the expression for $\mathrm{f}_{1}^{(j)}$, given in Equation (A.26), the quantities $\beta$ and $\beta^{2}-1$ appear, which in the limit of large $j$ have leading contributions of $\beta \propto j$ and $\beta^{2}-1 \propto j^{2}$. In this limit,

$$
\begin{aligned}
& f_{1}^{(j)}(\alpha)=\alpha \frac{\left(\begin{array}{c}
2 \beta D_{\alpha} b_{1 / 2}^{(j)}(\alpha)+j\left(3+\beta^{2}\right) b_{1 / 2}^{(j)}(\alpha) \\
-\alpha \delta_{j, 1}\left(\beta^{2}+2 \beta+3\right)
\end{array}\right)}{\beta^{2}\left(1-\beta^{2}\right)} \\
& f_{1}^{(j)}(\alpha) \rightarrow \alpha \frac{2 j^{2} \alpha^{j}+j\left(3+j^{2}\right) \alpha^{j}}{j^{4}} \\
& f_{1}^{(j)}(\alpha) \rightarrow \alpha \frac{j^{3} \alpha^{j}}{j^{4}}=\frac{\alpha^{j+1}}{j} .
\end{aligned}
$$

Similarly, for the outer planet, $\kappa \rightarrow j \alpha^{-3 / 2}$ and $1-\kappa^{2} \rightarrow j^{2} \alpha^{-3}$ so that

$$
\begin{aligned}
f_{2}^{(j)}(\alpha)= & -\left(j\left(\kappa^{2}+3\right) b_{1 / 2}^{(j)}(\alpha)+2 \kappa\left(b_{1 / 2}^{(j)}(\alpha)\right.\right. \\
& \frac{\left.\left.+D_{\alpha} b_{1 / 2}^{(j)}(\alpha)\right)-\alpha^{-2} \delta_{j, 1}\left(\kappa^{2}-2 \kappa+3\right)\right)}{\kappa^{2}\left(1-\kappa^{2}\right)} \\
f_{2}^{(j)}(\alpha) \rightarrow & \frac{j \kappa^{2} \alpha^{j}}{\kappa^{4}} \rightarrow \frac{\alpha^{j+3}}{j} .
\end{aligned}
$$

Therefore, systems with smaller values of $\alpha$ will have smaller synodic TTVs and, given $\alpha$, the terms with larger $j$ are less important in determining the TTVs.

\section{REFERENCES}

Agol, E., Steffen, J., Sari, R., \& Clarkson, W. 2005, MNRAS, 359, 567

Ballard, S., Fabrycky, D., Fressin, F., et al. 2011, ApJ, 743, 200

Batalha, N. M., Rowe, J. F., Bryson, S. T., et al. 2013, ApJS, 204, 24

Batygin, K., \& Morbidelli, A. 2013, AJ, 145, 1

Borucki, W. J., Koch, D. G., Basri, G., et al. 2011, ApJ, 736, 19

Carter, J. A., Agol, E., Chaplin, W. J., et al. 2012, Sci, 337, 556

Dreizler, S., \& Ofir, A. 2014, arXiv: 1403.1372

Ferraz-Mello, S., Michtchenko, T. A., \& Beaugé, C. 2005, ApJ, 621, 473

Fischer, D. A., Schwamb, M. E., Schawinski, K., et al. 2012, MNRAS, 419, 2900

Foreman-Mackey, D., Hogg, D. W., Lang, D., \& Goodman, J. 2013, PASP, 125,306

Gladman, B. 1993, Icar, 106, 247

Hadden, S., \& Lithwick, Y. 2013, arXiv:1310.7942

Hamilton, D. P. 1994, Icar, 109, 221

Hansen, B. M. S., \& Murray, N. 2013, ApJ, 775, 53

Hansen, B. M. S., \& Murray, N. 2014, arXiv:1405.2342

Holman, M. J., \& Murray, N. W. 2005, Sci, 307, 1288

Holman, M. J., Fabrycky, D. C., Ragozzine, D., et al. 2010, Sci, 330, 51

Huber, D., Carter, J. A., Barbieri, M., et al. 2013, Sci, 342, 331

Lissauer, J. J., Fabrycky, D. C., Ford, E. B., et al. 2011, Natur, 470, 53

Lissauer, J. J., Jontof-Hutter, D., Rowe, J. F., et al. 2013, ApJ, 770, 131

Lithwick, Y., \& Wu, Y. 2012, ApJL, 756, L11

Lithwick, Y., Xie, J., \& Wu, Y. 2012, ApJ, 761, 122

Mahajan, N., \& Wu, Y. 2014, arXiv:1409.0011

Masuda, K. 2014, ApJ, 783, 53

Migaszewski, C., Goździewski, K., \& Słonina, M. 2013, MNRAS, 436, L25

Miralda-Escudé, J. 2002, ApJ, 564, 1019

Murray, C. D., \& Dermott, S. F. 1999, Solar System Dynamics (Cambridge: Cambridge Univ. Press)

Nesvorný, D. 2009, ApJ, 701, 1116

Nesvorný, D., \& Beaugé, C. 2010, ApJL, 709, L44

Nesvorný, D., Kipping, D., \& Terrell, D. 2014, arXiv:1405.2060

Nesvorný, D., Kipping, D., Terrell, D., et al. 2013, ApJ, 777, 3

Nesvorný, D., Kipping, D. M., Buchhave, L. A., et al. 2012, Sci, 336, 1133

Nesvorný, D., \& Morbidelli, A. 2008, ApJ, 688, 636

Nesvorný, D., \& Vokrouhlický, D. 2014a, ApJ, 796, 148

Nesvorný, D., \& Vokrouhlický, D. 2014b, ApJ, 790, 58

Schmitt, J. R., Agol, E., Deck, K. T., et al. 2014, ApJ, 795, 167

Schneider, J. 2003, SF2A-2003: Semaine de l'Astrophysique Française ed. F. Combes et al. (Les Ulis: EDP Sciences), 149

Tenenbaum, P., Jenkins, J. M., Seader, S., et al. 2013, ApJS, 206, 5

Vokrouhlický, D., \& Nesvorný, D. 2014, ApJ, 791, 6

Xie, J.-W., Wu, Y., \& Lithwick, Y. 2014, ApJ, 789, 165 